\title{
The LEAFY floral regulator displays pioneer transcription factor
}

4 Xuelei Lai ${ }^{1, *}$, Romain Blanc-Mathieu ${ }^{1, *}$, Loïc GrandVuillemin ${ }^{1, \wedge}$, Ying Huang ${ }^{2, \wedge}$, Arnaud

5 Stigliani ${ }^{1,3}$, Jérémy Lucas ${ }^{1}$, Emmanuel Thévenon ${ }^{1}$, Jeanne Loue-Manifel ${ }^{1,4}$, Hussein Daher ${ }^{1,5}$,

6 Eugenia Brun-Hernandez ${ }^{1}$, Gilles Vachon ${ }^{1}$, David Latrasse ${ }^{2}$, Moussa Benhamed ${ }^{2,6}$, Renaud

7 Dumas $^{1}$, Chloe Zubieta ${ }^{1}$ and François Parcy ${ }^{1, * *}$

9 'Laboratoire Physiologie Cellulaire et Végétale, Univ. Grenoble Alpes, CNRS, CEA, INRAE,

10 IRIG-DBSCI-LPCV, 17 avenue des martyrs, F-38054, Grenoble, France

11 2Université Paris-Saclay, CNRS, INRAE, Univ. Evry, Institute of Plant Sciences Paris-Saclay

12 (IPS2), 91405, Orsay, France.

$13{ }^{3}$ The Bioinformatics Centre, Department of Biology and Biotech and Research Innovation

14 Centre, University of Copenhagen, Ole Maaløes Vej 5, DK2200 Copenhagen N, Denmark

$15{ }^{4}$ Laboratoire Reproduction et Développement des Plantes, Université de Lyon, ENS de Lyon,

16 UCB Lyon 1, CNRS, INRA, F-69342 Lyon, France

$17{ }^{5}$ Institut de Biologie Structurale, Université Grenoble Alpes, CEA, CNRS, Grenoble, France

$18{ }^{6}$ Université de Paris, Institute of Plant Sciences Paris-Saclay (IPS2), F-75006 Paris, France

$20 *$ contributed equally

$21 \wedge$ contributed equally to the work

$22 * *$ Correspondence: François Parcy (francois.parcy@cea.fr) 


\section{Abstract}

24 Pioneer transcription factors (TFs) are a special category of TFs with the capacity to bind to 25 closed chromatin regions in which DNA is wrapped around histones and often highly 26 methylated. Subsequently, they are able to modify the chromatin state to initiate gene 27 expression. In plants, LEAFY (LFY) is a master floral regulator and has been suggested to act 28 as a pioneer TF in Arabidopsis. Here, we demonstrate that LFY is able to bind both methylated 29 and non-methylated DNA using a combination of in vitro genome-wide binding experiments 30 and structural modeling. Comparisons between regions bound by LFY in vivo and chromatin 31 accessibility data suggest that LFY binds a subset of regions occupied by nucleosomes. We 32 confirm that LFY is able to bind nucleosomal DNA in vitro using reconstituted nucleosomes. 33 Finally, we show that constitutive LFY expression in seedling tissues is sufficient to induce 34 chromatin accessibility in the LFY direct target genes, APETALA1 and AGAMOUS. Taken 35 together, our study suggests that LFY possesses key pioneer TF features that contribute to launch the floral gene expression program. 


\section{Introduction}

Proper gene regulation is essential to all living organisms, controlling processes from basic development to environmental response. Gene regulation requires the finely orchestrated activity of transcription factors (TFs) that recognize specific DNA sequences in gene regulatory regions and activate or repress transcription of their target genes. While the binding of most TFs to DNA is restricted to accessible regions of the genome, a specific type of TF, called a "pioneer", is able to access its cognate binding site even in closed, nucleosome-rich chromatin regions (Magnani et al., 2011; Iwafuchi-Doi and Zaret, 2014; Iwafuchi-Doi and Zaret, 2016; Zaret, 2020). The ability to bind nucleosomal DNA in vivo and in vitro is a defining characteristic of pioneer TFs and has been well-established for diverse mammalian pioneer TFs (Fernandez Garcia et al., 2019). As DNA in closed chromatin regions is often highly methylated, another emerging feature of pioneer TFs is their capability to bind DNA in a methylation insensitive manner (Zhu et al., 2016; Mayran and Drouin, 2018). Some pioneer TFs are even able to directly recruit DNA demethylases at methylated sites, thereby facilitating the remodeling of closed regions (Iwafuchi-Doi, 2018).

Pioneer TFs are often master regulators controlling developmental transitions, with the mammalian pluripotency factors OCT4, SOX2, and KLF4 representing some of the most wellstudied (Soufi et al., 2015). These factors bind to closed chromatin regions and induce their opening or remodeling, so that genes they contain can be activated by the pioneer TFs themselves or by other TFs called settlers (Sherwood et al., 2014; Slattery et al., 2014). The modification of the chromatin landscape by pioneer TF can be accomplished either directly by triggering DNA detachment from nucleosomes (Dodonova et al., 2020; Michael et al., 2020), or indirectly by the recruitment of ATP-dependent cellular machineries, such as chromatin remodelers that remove or modify adjacent nucleosomes in order to prime downstream regulatory events (Hu et al., 2011; King and Klose, 2017). Such capacity to modify DNA accessibility is another defining feature of pioneer TFs (Iwafuchi-Doi and Zaret, 2014).

In plants, the only TF reported as pioneer TF so far is LEAFY COTYLEDON1 (LEC1), a seed specific TF involved in embryonic epigenetic reprogramming (Tao et al., 2017). LEC1 was shown to promote the initial establishment of an active chromatin state of its target gene in silenced chromatin and activate its expression de novo. Pioneer TF activity was also suggested for two types of factors controlling flower development, the MADS homeotic TFs (Pajoro et al., 2014; Denay et al., 2017) and the master floral regulator, LEAFY (LFY). The MADS TFs, 
including APETALA1 (AP1) and SEPALLATA3, were shown to be able to access closed chromatin regions to specify floral organs, and were thus postulated to act as pioneer TFs (Pajoro et al., 2014). However, mammalian MADS TFs do not seem to act as pioneer factors and thus the identification of AP1 and SEP3 as potential pioneers remains speculative (Sherwood et al., 2014). In contrast to the MADS TFs, one previous study suggest that LFY may have pioneer activity (Sayou et al., 2016). LFY is a master regulator specifying the floral identity of meristems. It directly induces the floral homeotic genes AP1, APETALA3 (AP3) and AGAMOUS (AG) (Parcy et al., 1998; Wagner, 1999; Lohmann et al., 2001; Chae et al., 2008; Yamaguchi et al., 2013; Chahtane et al., 2013). $A G$ and $A P 3$ are known to be under the repression of Polycomb repressive complexes in seedlings (Goodrich et al., 1997; Turck et al., 2007; Calonje et al., 2008). This suggests that their activation during flower development requires modifications of their chromatin landscape and that the direct binding of LFY to their regulatory regions might trigger. Consistent with this, LFY was suggested to be able to access closed chromatin regions in vivo (Sayou et al., 2016). Moreover, LFY's role is not confined to conferring a flower fate to meristems. It can also contribute to meristem emergence (Moyroud et al., 2010; Chahtane et al., 2013; Yamaguchi et al., 2013), and together with its co-regulators such as the homeodomain TF WUSCHEL or the F-Box protein UNUSUAL FLORAL ORGANS, it can even induce meristem formation from root or leaf tissue, respectively (Levin and Meyerowitz, 1995; Gallois et al., 2004; Risseeuw et al., 2013). Taken together, these data indicate that LFY has the full capability of reprogramming cell fate, a property often requiring pioneer activity. However, whether LFY is truly able to directly bind closed chromatin regions and change their status has yet to be demonstrated.

Here, we address the pioneer activity of LFY in vitro and in vivo. Firstly, we determined whether LFY binding was sensitive to DNA methylation. For this, we combined in vitro LFY genome-wide binding data using methylated and unmethylated genomic DNA and structural analysis. These experiments demonstrated that LFY binding is only mildly sensitive to DNA methylation. In order to test whether LFY binding was compatible with the presence of nucleosomes, we compared LFY binding data from chromatin immunoprecipitation sequencing (ChIP-seq) and chromatin accessibility data. Based on these comparisons, we found that LFY could access a number of closed chromatin regions and that LFY colocalizes with nucleosomes in some regions in vivo. Using electrophoretic mobility shift assays (EMSA), we further showed that LFY was able to directly bind nucleosomes in vitro. Finally, chromatin accessibility assays demonstrated that LFY constitutive expression was sufficient to increase chromatin 
accessibility in genomic regions including its known target genes $A P l$ and $A G$. Taken together, these data establish that LFY is able to act as a pioneer TF in the regulation of important target genes critical for the establishment of floral fate.

\section{Results}

\section{LFY is weakly sensitive to DNA methylation}

In closed chromatin regions, DNA is packed within nucleosomes (McGinty and Tan, 2015) and its level of methylation is often higher than in open chromatin (Chodavarapu et al., 2010). Both the presence of nucleosome and DNA methylation usually reduce TFs access and their binding to target DNA (Yin et al., 2017; Klemm et al., 2019). In order to assess the effect of methylation on LFY binding to DNA, we applied DNA Affinity Purification sequencing (DAP-seq) (O'Malley et al., 2016). Similar to ChIP-seq, this technique allows the identification of the genomic regions bound by a TF but using naked DNA and a recombinant TF. We used Arabidopsis genomic DNA extracted from seedlings that was either PCR amplified (ampDAP, DNA cleared of methylation) or not amplified (DAP, DNA retaining methylation). Both experiments were performed in triplicates with high reproducibility (Supplemental Figure 1; Supplemental Table 1). As controls, we used two TFs described as methylation sensitive based on available ampDAP and DAP datasets (O’Malley et al., 2016) (Supplemental Figure 2). For each genomic region bound by a given $\mathrm{TF}$, we plotted the DAP/ampDAP signal ratio as a function of the methylation density in the whole bound region (based on Arabidopsis seedling methylation maps (Zhang et al., 2016)). If the DNA binding of a TF is inhibited by methylation, we expect the DAP/ampDAP ratio to decrease when the methylation level increases. LFY DNA binding was much less affected by increasing methylation density than the two methylation sensitive TFs, (Figure 1A-C). To analyze more precisely the effect of methylation, we tested the correlation between the number of methylated cytosines within the best TF binding (TFBS) site, identified using position weight matrices in each bound region and the DAP/ampDAP ratio of bound regions. Whereas an increased number of methylated cytosines in TFBS strongly decreased the binding for the two methylation sensitive TFs in DAP relative to ampDAP, LFY binding was only mildly affected (Figure 1D-F). Finally, we designed a specific procedure to compute the effect of methylation on each individual cytosine possibly present in the best TFBS (Supplemental Figure 3-5). In the case of LFY, we identified two positions where the binding is increased by cytosine methylation (positions 4 on the forward DNA strand and 5 on the reverse), and other positions (2,3,7,8 on the forward strand and 1,3,4,9 on the reverse) where 
134 the binding is only mildly inhibited (Figure $1 \mathrm{G}$ ). In contrast, methylation was inhibitory for the

135 two methylation sensitive TFs in most positions where a cytosine can possibly be present

136 (Figure 1H-I). Structural analysis of LFY DNA binding domain in complex with DNA (Hamès

137 et al., 2008) provided a biochemical explanation of these positive and negative effects

138 (Supplemental Figure 6). In particular, the hydrophobic contacts between LFY and DNA are

139 likely to be enhanced by the presence of a methyl group in positions 4 and 5 of the LFY binding 140 site (LFYBS), consistent with the DAP versus ampDAP analysis (Figure 1G).
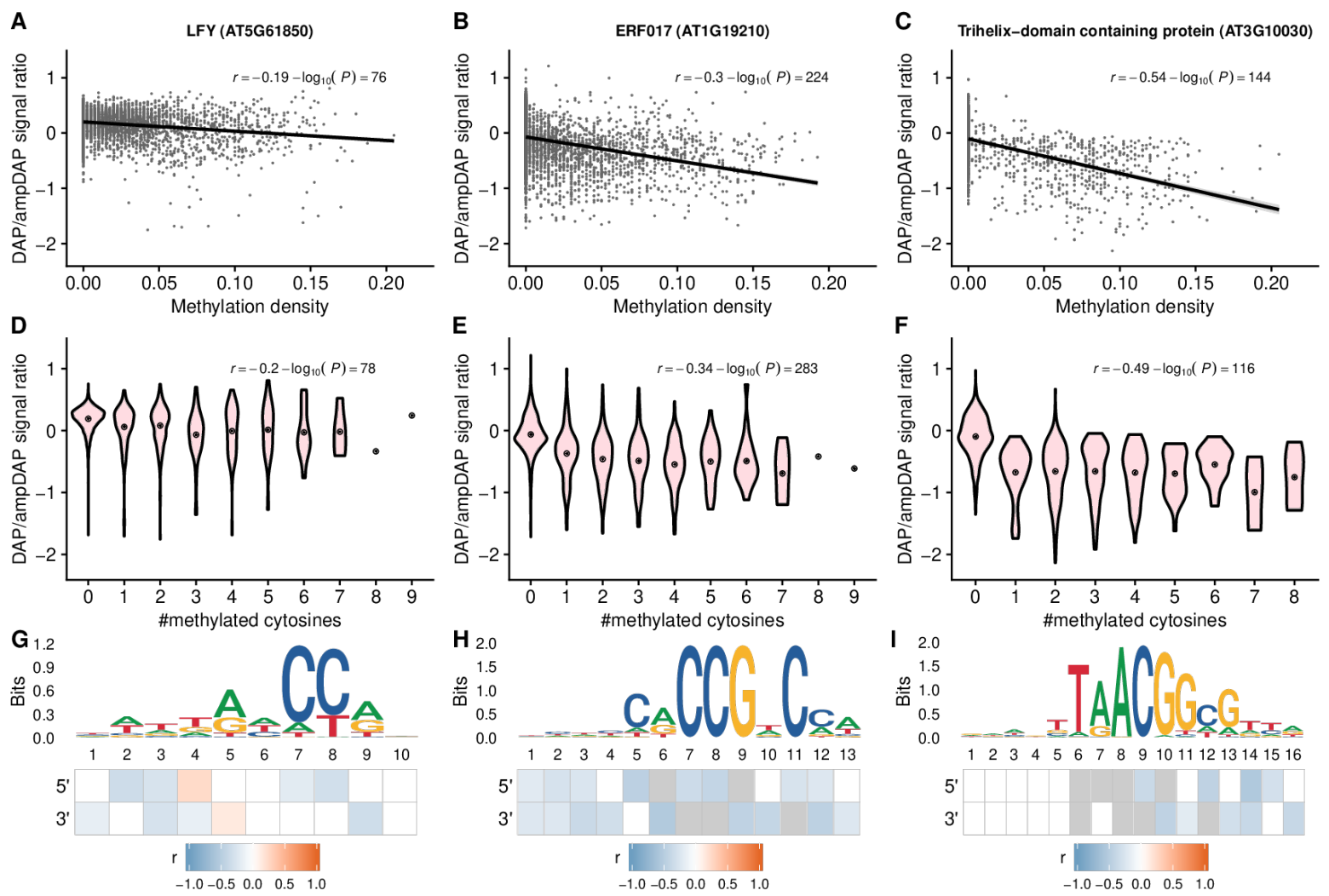

Figure 1: Cytosine methylation has a mild effect on LFY DNA-binding intensity.

144 Effect of cytosine methylation on DNA binding for three transcription factors: LFY (left),

145 ERF017 (middle) and a trihelix-domain containing protein (right). (A-C) Biplots between the

146 DAP/ampDAP signal ratio (peak normalized read coverage in the DAP experiment divided to

147 that in the ampDAP experiment) in a $\log _{10}$ scale and methylation density (proportion of

148 cytosines with a probability of methylation greater than 0.5 ) within transcription factor bound

149 regions. The increasing methylation density has weaker effect on LFY than on the two other

150 TFs. (D-F) Violin plots of DAP/ampDAP signal ratio in a $\log _{10}$ scale as a function of the number

151 of methylated cytosines in the best TF binding site (TFBS) of each bound region. LFY binding

152 is barely affected by the increased number of methylated cytosines. (G-I) Binding site sequence 
153 motif for each TF and the methylation effect on each individual position. For LFY, a single half

154 of the symmetric motif is shown. Heatmaps show the Pearson's correlation coefficient $(r)$ 155 between the DAP/ampDAP signal ratio in a $\log _{10}$ scale and the probability of methylation at 156 each position of the best TFBSs. Blank positions have a high false discovery rate (> 5\%) and 157 grey indicates positions with less than ten cytosines in the dataset. Correlation are tested on 158 both sides of a symmetric motif $(\mathrm{G})$ or on both strands for non-symmetric motifs (H-I).

\section{LFY binds to a subset of the closed chromatin regions}

160 Next, we analyzed how in vivo factors (including the chromatin state) affect LFY DNA binding. 161 For this, we compared LFY binding in vitro and in vivo by plotting the coverage of LFY DAP162 seq peaks versus that of LFY ChIP-seq peaks. LFY ChIP-seq was obtained from $35 S:: L F Y$ seedlings or floral meristems (Sayou et al., 2016; Goslin et al., 2017). This analysis identified genome regions well bound in both experiments (Figure 2A; Supplemental Figure 7A; colored in light purple to red). However, it also highlighted the existence of regions much better bound in vivo (ChIP-specific regions colored in deep purple) or in vitro (DAP-specific regions colored in orange). The existence of ChIP-specific regions indicated that LFY DNA binding might increase due to interactions with in vivo factors. The presence of DAP-specific regions indicated that the in vivo context inhibits LFY from binding to some genomic regions despite high affinity LFY binding sites are observed in those regions in DAP-seq.

171 To understand whether chromatin conformation could play a role in this inhibition, we analyzed

172 the chromatin state of each region using DNaseI-seq data obtained in two-week-old seedlings

173 (Zhang et al., 2012), a high DNaseI-seq signal being indicative of an open region (Figure 2B; 174 Supplemental Figure 7B). We found that many of the DAP-specific regions have a low DNaseI 175 signal, typical of closed chromatin regions. This suggests that closed chromatin regions inhibit 176 LFY binding. However, as previously observed (Sayou et al., 2016), a number of regions are bound in ChIP-seq despite low DNaseI signal (right panels on Figure 2B and Supplemental

178 Figure 7B). Overall, this analysis suggests that while the closed chromatin context is generally 179 inhibitory for LFY binding, some closed chromatin regions can still be bound. To analyze what 180 type of closed regions are most likely to be bound, we analyzed the upper and lower deciles of 181 regions ranked based on their ChIP-seq signal, the upper decile contains regions well bound in 182 ChIP whereas the lower has regions poorly bound in ChIP (but bound in DAP). The distribution 183 of nine chromatin states (as defined in the literature (Sequeira-Mendes et al., 2014)) changes 184 drastically between the two deciles (Figure 2C; Supplemental Figure 7C). Chromatin states 7, 1858 , and 9 (that is the most compacted and includes heterochromatin) are unlikely to be bound 
186 whereas states 1-5, which represent closed regions but closer to gene units or targets of

187 Polycomb repression (state 5) are more frequently found in LFY bound regions, showing that 188 closed regions are not all equivalently contacted by LFY depending on their functional 189 category.

190 As closed chromatin regions are often occupied by nucleosomes, and since in vivo data suggests

191 that LFY might be able to bind some of these regions, we wondered whether LFY binding was 192 compatible with the presence of nucleosomes. To test this, we compared the position of LFY 193 ChIP-seq peaks with that of nucleosomes (based on MNase-seq data (Zhang et al., 2015)). We

194 found that nucleosomes were indeed enriched at the center of LFY ChIP-seq peaks in closed 195 regions (Figure 2D; Supplemental Figure 7D), but not in open ones (Figure 2E; Supplemental 196 Figure 8E), suggesting that LFY might be able to directly bind nucleosomal DNA in vivo. The 197 mapping of LFY TFBS in nucleosome-occupied LFY ChIP-seq peaks show a slight enrichment 198 at the center of the nucleosome, around the dyad position which is a site commonly bound by 199 pioneer TFs (Figure 3A; Supplemental Figure 8) (Zaret, 2020). However, since these genomic 200 data are established on mixtures of tissues, they are not sufficient to firmly establish that LFY 201 is indeed able to bind nucleosomal DNA.
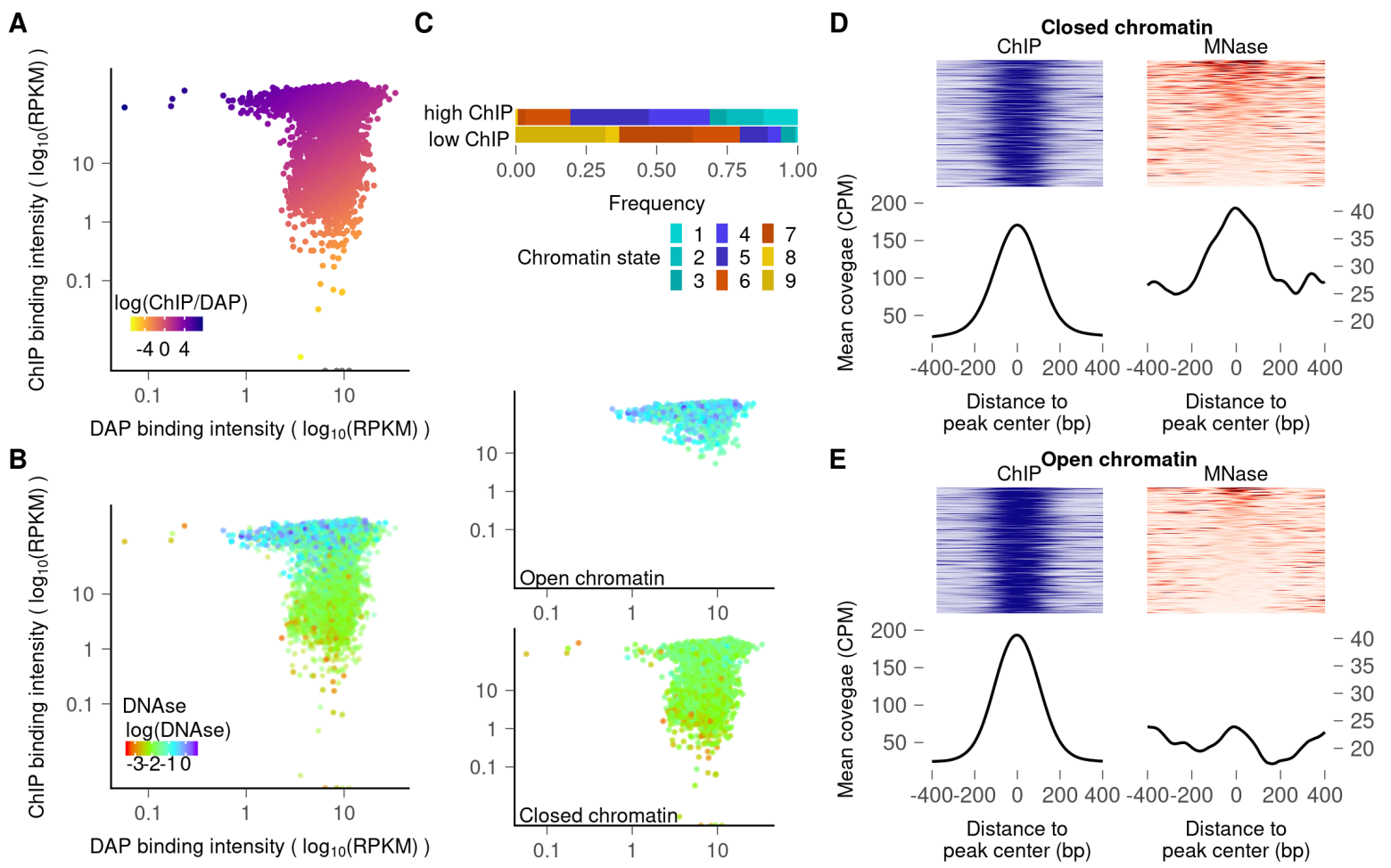

Figure 2: LFY is able to bind nucleosomes in closed chromatin regions.

204 (A) Plots comparing the LFY binding intensities in ChIP-seq vs DAP-seq experiments. Heat map is based on the ChIP-seq/DAP-seq intensity ratio. (B) Overlay of DNaseI signal (heat map) 
on LFY bound regions, with DAP-seq (X-axis) and ChIP-seq (Y-axis) peak coverages. The two panels on the right show the same regions split into open (upper panel) and closed (lower panel) chromatin states. (C) Distribution of chromatin states 1 to 9 according to (Sequeira-Mendes et al., 2014) for the first and last decile of LFY bound regions based on ChIP-seq signal. (D-E) MNase signal around ChIP-seq peak centers in closed (D) or open (E) chromatin regions. Upper panels show ChIP-seq and MNase-seq coverage for each peak ordered based on MNase-seq signal. Lower panels represent the mean coverage.

\section{LFY binds nucleosomal DNA at specific sites in vitro}

214 Next, we tested whether LFY has the capacity to bind nucleosomal DNA in vitro. We first assembled nucleosomes using the Widom 601 strong nucleosome positioning sequence (Lowary and Widom, 1998; McGinty and Tan, 2015), in which a LFY binding site (LFYBS) was inserted at different positions (C1-C7 around the dyad and E1-E7 farther away) (Figure 3B; Supplemental Table 2). Nucleosomes assembled with a LFYBS at position C2 and C7 were shifted upon addition of LFY, whereas no shift was observed for nucleosomes with a LFYBS at positions C1, C3-C6, E1-E7 or with no LFYBS, demonstrating that LFY binds nucleosomal DNA in a sequence specific manner and only with a LFYBS present at specific positions $(\mathrm{C} 2$, located around the dyad, and C7, located one helix turn apart from C2, with the LFYBS exposed to the outer nucleosome surface (Figure 3B and C; Supplemental Figure 9)). Using the same methodology, as a negative control, we tested nucleosomal DNA binding of the TF REGULATOR OF AXILLARY MERISTEMS 1 (RAX1), a direct downstream target of LFY (Chahtane et al., 2013). We found that RAX1 cannot associate with nucleosomes even when its binding site is exposed to the outer nucleosome surface and at the dyad (Supplemental Figure 10), suggesting that RAX1 is unlikely a pioneer TF. We also assembled nucleosomes with two regions of the $A P 1$ gene, a known early activated LFY target (Parcy et al., 1998; Wagner, 1999; Benlloch et al., 2011). These regions were taken from $A P 1$ first intron and $A P 1$ promoter 231 (annotated as APl intron and APl pro, respectively, in Figure 3D). They are both bound by 232 LFY in vivo (ChIP-seq (Moyroud et al., 2011; Winter et al., 2011; Sayou et al., 2016; Goslin et 233 al., 2017)) and in vitro (DAP-seq in Figure 3D), and with well-defined nucleosome signals from 234 MNase-seq in both seedlings and flower tissues (Zhang et al., 2015) (Figure 3D). We observed 235 that LFY was able to bind to these nucleosomes (Figure 3E and Supplemental Figure 11), showing that LFY nucleosomal DNA binding also occurs within Arabidopsis genomic regions. 
A

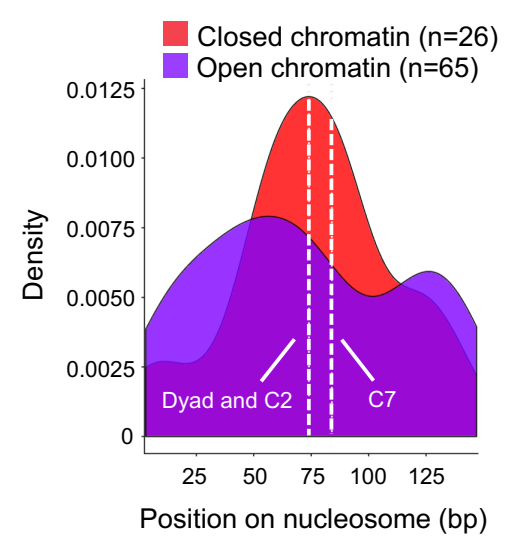

C

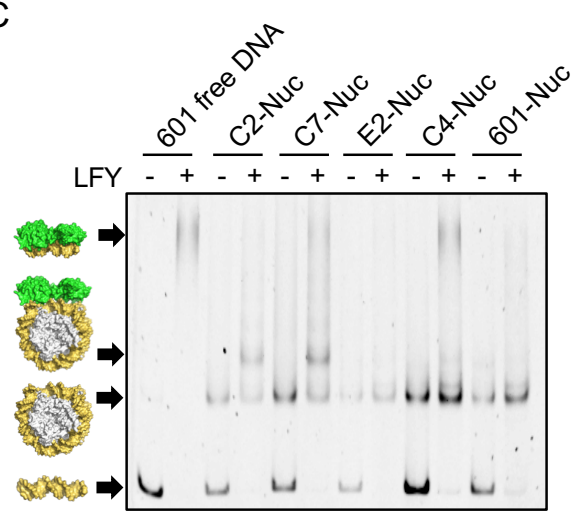

B

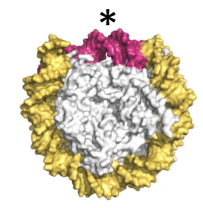

C1-Nuc

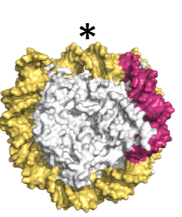

E1-Nuc

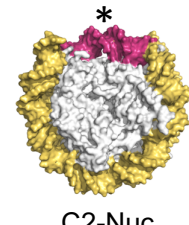

C2-Nuc

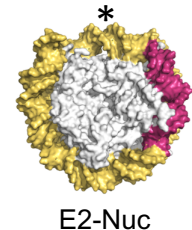

LFYBS shift every 2 bp clock-wise
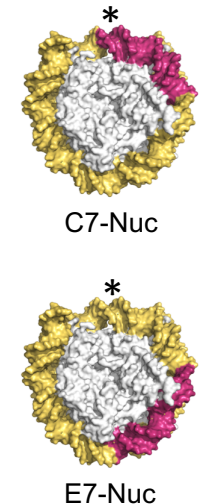

E

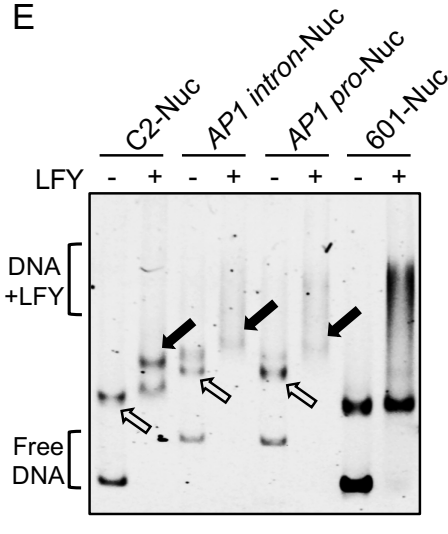

Figure 3: LFY binds nucleosomes in vitro

239 (A) Density plot of the LFY best binding site along a canonical 147-bp nucleosomal sequence 240 in open and closed chromatin contexts for flower tissues. An enrichment for LFY binding sites

241 (LFYBS) around the dyad position (the center of the nucleosomal DNA) is observed in closed 242 chromatin regions. C2 (at dyad) and C7 positions are indicated. Alternative plots for different 243 thresholds for binding sites selection are reported in Supplemental Figure 8. (B) Design of 244 Widom 601 sequences (yellow orange) with a LFYBS (warm pink) inserted at different 245 positions (central C1-C7 (top) and external E1-E7 (bottom)) on nucleosome (PDB: 3UT9 (Chua et al., 2012). * indicates the dyad. (C) Representative EMSA showing LFY binding to 601 nucleosomes with a LFYBS at positions C2 (labelled C2-Nuc) and C7, but not at E2, C4 or 601 nucleosome without a LFYBS (refer to Supplemental Figure 9 for the screening of LFY nucleosomal DNA binding at all other positions). Free DNA (C2 in the first 2 lanes, or present in the nucleosomal preparations) is shifted at the very top of the gels. 601-Nuc is made with wild-type 601 sequence (without a LFYBS): only free DNA is shifted due to non-sequence specific interactions with LFY. Cartoon on the side from bottom to top are free DNA, nucleosome alone, LFY-nucleosome complex and free DNA-LFY complex. (D) Genomic 
snapshots of LFY DAP-seq, ChIP-seq (seedlings tissue), and MNase-seq (seedlings and closed

255 flower buds) at the $A P 1$ loci. $A P 1$ intron and $A P 1$ pro sequences used to assemble nucleosomes in (E) are highlighted in grey. Both regions are bound in DAP and ChIP, and with well-defined nucleosome signals, lower in floral tissue than in seedlings. (E) EMSA showing LFY binding to nucleosomes assembled with $A P 1$ intron and $A P 1$ pro sequences. AP1-intron-Nuc and AP1pro-Nuc are longer than 601 due to the presence of amplification primers. Note some free 601 DNA is shifted despite the absence of LFYBS in the last lane. The hollow and solid arrows indicate the position of reconstituted nucleosomes and the shifted nucleosomes signals, respectively.

\section{LFY constitutive expression induce changes in nucleosome position}

One key characteristic feature of a pioneer TF is to be able to modify the status of closed chromatin regions (Iwafuchi-Doi and Zaret, 2014). To test whether LFY is able to do so, we examined whether it could alter nucleosome positions when ectopically expressed in seedlings. We selected regions that are closed in wild-type seedlings and with a mapped nucleosome (Zhang et al., 2015) and bound by LFY in ChIP-seq and DAP-seq (Figure 4) (Sayou et al., 2016). Among these regions, we focused on the three floral regulators, $A P 1$ and $A G$ (two established LFY targets), and ULTRAPETALA 1 (ULT1), another floral regulator involved in $A G$ activation (Moreau et al., 2016). Using Formaldehyde-Assisted Isolation of Regulatory Elements (FAIRE)-qPCR that identifies nucleosome depleted regions, we tested whether ectopic LFY expression ( $35 S: \because L F Y)$ could alter the local chromatin as compared to two-weekold Col-0 seedlings where endogenous LFY is not yet highly induced. We found that LFY ectopic expression induced nucleosome depletion in these regions (Figure 4A). Interestingly, in the $A P 1$ intron, a region strictly inaccessible in Col-0 seedlings according to DNAseI signal, LFY expression triggers a strong depletion (3-fold, position P1 in Figure 4A). In the AP1 promoter, a region already largely accessible, LFY induced depletion is more moderate $(\mathrm{P} 2$, P3; Figure 4A). As controls, we tested three regions (Actin2, AT2G38220 and AT4G22285) where LFY does not bind in vivo and in vitro and with poor accessibility in seedlings. We found that their nucleosome status was not altered by LFY ectopic expression (Figure 4B). Taken together, our data suggests that LFY ectopic expression in seedling tissues is sufficient to trigger nucleosome depletion in regulatory regions of some key floral regulators including two LFY target genes. 
A
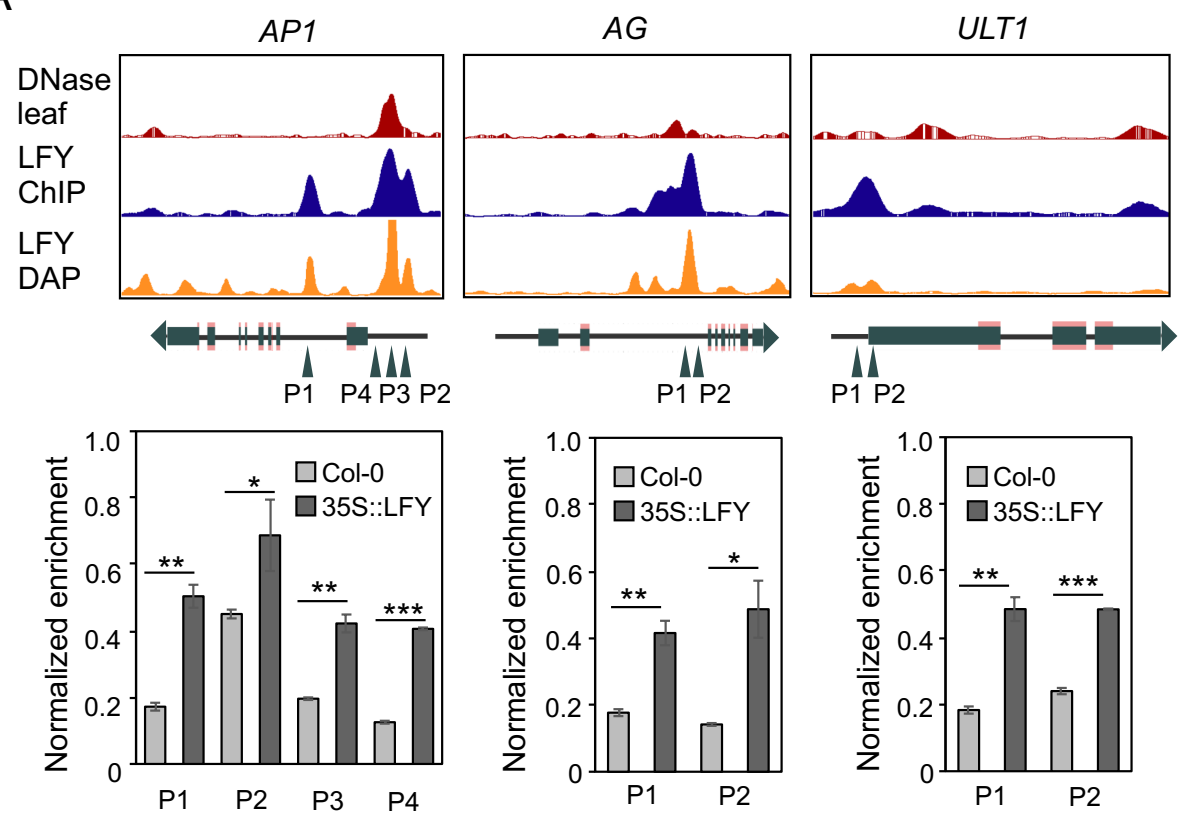

B
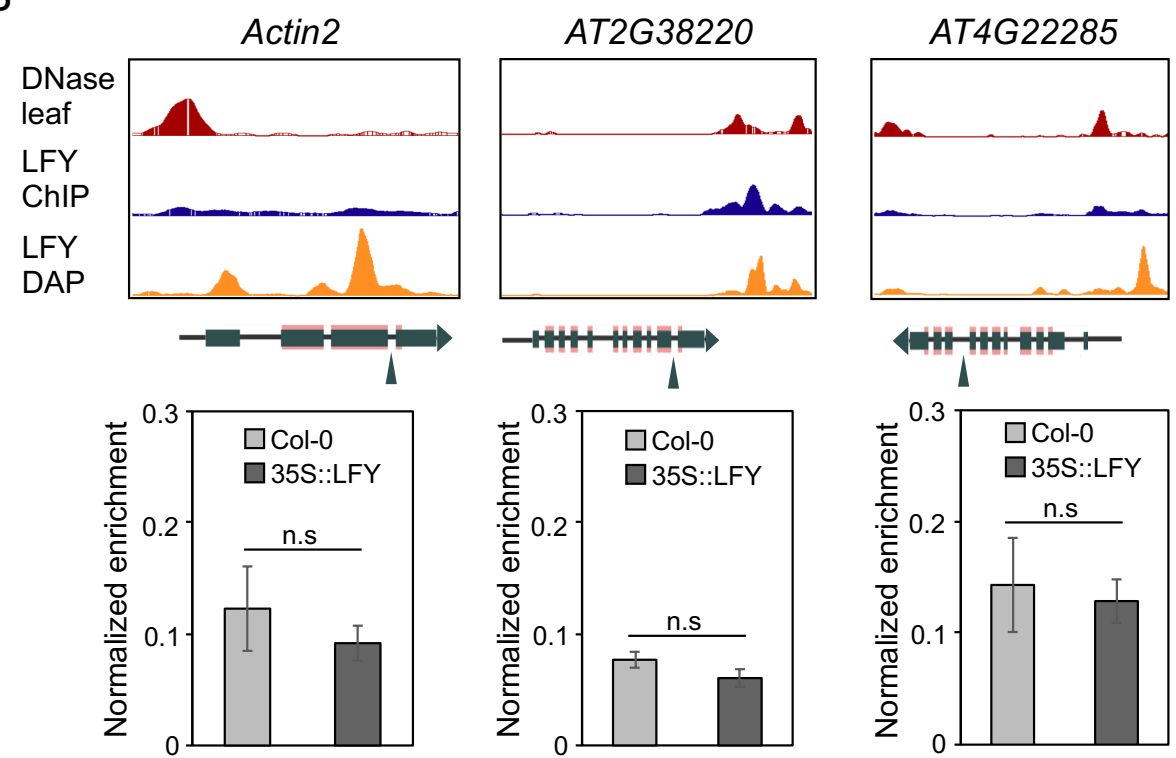

286 Figure 4: LFY constitutive expression increases chromatin accessibility.

287 (A) (Top) Genomic snapshots of chromatin accessibility (DNaseI-seq from 2-week-old Col-0 288 seedlings (Zhang et al., 2012)), LFY binding in vitro (DAP-seq using genomic DNA from 2289 week-old $35:: L F Y$ seedlings), in vivo (ChIP-seq of 2-week-old $35:: L F Y$ seedlings (Sayou et al., 2016)) at $A P 1, A G$ and $U L T 1$ loci. The regions tested in FAIRE-qPCR are indicated by triangle

291 arrows. (Bottom) FAIRE-qPCR of the indicated regions are performed in 2-week-old seedlings 292 of Col-0 (pale gray) and $35 S:: L F Y$ (dark gray), respectively. Error bars represent means \pm 293 standard deviation. Significance test is performed by one-tailed students' t-test, ${ }^{*} \mathrm{p}<0.05$, $294 * * \mathrm{p}<0.01, * * * \mathrm{p}<0.001$, n.s, not significant. (B) (Top) genome browser snapshots of three 
genomic regions devoid of LFY binding and poorly accessible in 2-week-old seedlings.

296 (Bottom) FAIRE-qPCR on the indicated regions. Significance test is performed as per (A). The 297 FAIRE-qPCR is performed by two biological replicates, with three technical replicates for each.

298 The enrichment is normalized by input DNA in each experiment.

\section{Discussion}

300 LFY is a master regulator of floral development able to induce the expression of floral organ 301 identity genes that are known to be under repression of Polycomb repressive complex 302 (Goodrich et al., 1997; Turck et al., 2007; Calonje et al., 2008; Kaufmann et al., 2010). In a 303 previous study (Sayou et al., 2016), ChIP-seq data suggested LFY could bind to closed

304 chromatin regions and possibly act as a pioneer factor, although a mechanism was never fully 305 described. By analyzing seedlings constitutively expressing LFY, it was suggested that LFY 306 wild type could bind to regions with a closed chromatin status and that this capacity was 307 strongly impaired upon mutation of the LFY oligomerization sterile alpha motif (SAM) domain 308 (Sayou et al., 2016). Oligomerization would likely increase the DNA-binding affinity of LFY, 309 as has been shown for other TFs able to multimerize (Lai et al., 2020). This increase in DNA 310 binding affinity may be critical for the efficient binding to partially occluded sites in closed 311 chromatin regions, however an increase in binding affinity alone is likely not sufficient to 312 enable recognition of a TFBS in a closed region of chromatin. Insensitivity to methylation state 313 and the presence of nucleosomes are prerequisites to pioneer function, which we investigated 314 here.

315 Given the high level of DNA methylation found in closed chromatin, it has been hypothesized 316 that some pioneer TFs would be able to bind DNA independently of its methylation status (Zhu 317 et al., 2016; Mayran and Drouin, 2018). Indeed, pioneer TFs from animals, such as Pax7 318 (Mayran et al., 2018, 7), OCT4 (Yin et al., 2017) and KLF4 (Hu et al., 2013; Liu et al., 2014), 319 are either insensitive or even prefer methylated DNA. The DNA binding of most TFs is 320 inhibited by DNA methylation because the 5-methyl group of methylcytosine often clashes 321 with protein residues that are involved in specific base readout (Yin et al., 2017). Some TFs, 322 however, are not sensitive or even favor methylated DNA because direct hydrophobic 323 interactions form between the methyl group and the TF, as it is the case for homeodomain TFs 324 (Yin et al., 2017) or for some basic leucine zipper TFs (Weber et al., 2019). In this study, we 325 showed that LFY is only mildly sensitive to methylation. We further dissected how methylation 326 impacts LFY binding for each individual position of a canonical LFY binding site (Figure 1). 
Consistent with our structural analysis, we showed that the key protein-DNA interactions are not affected by cytosine methylation (Supplemental Figure 6), and even that, at two positions, a methyl group might enhance LFY binding. This computational analysis has the potential to be generalized to all TFs for which DAP and ampDAP data are available. It represents a powerful complement to methylation-sensitive SELEX (systematic evolution of ligands by exponential enrichment) analysis which was used to detect the effect of methylation to TFDNA binding using randomized DNA sequences (Yin et al., 2017).

Next, we examined whether LFY could bind nucleosomal DNA in vivo. Overall, for the majority of regions, a closed chromatin state has inhibitory effect on LFY binding. This is particularly true for heterochromatin regions likely because their high level of compaction totally prevents TF access. However, a subset of closed regions present in the vicinity of genes showed a LFY binding signal in ChIP-seq both in seedlings and floral tissues, consistent with previous observations (Sayou et al., 2016). The limitation of such prior analysis is the heterogeneity of the tissue used: it is conceivable that the observed LFY binding signal would come from the subset of cells where the regions are open. Using in vitro reconstituted nucleosomes, we show here that LFY has the capacity to bind nucleosomal DNA. This property is consistent with structural data showing that LFY binds a single side of the DNA and with assays where LFYBS position was either exposed to the outer surface (like C2 or C7) or partially hidden by histones ( $\mathrm{C} 1, \mathrm{C} 3$ to $\mathrm{C} 6)$. Moreover, we found that $L F Y$ ectopic expression was able to increase nucleosome depletion at several target loci. We examined in particular the AP1 gene, a known direct target of LFY (Parcy et al., 1998; Wagner, 1999) that is induced immediately after LFY during flower development. LFY binds to two $A P 1$ regions, its promoter and its first intron. According to DNAseI signal, $A P 1$ promoter is a region already open in seedlings and with two nucleosomes detected by MNase-seq. This observation likely explains that $A P 1$ promoter can be induced by LFY already in seedlings and independently of flower formation (Parcy et al., 1998). Here, we observe that LFY ectopic expression is able to induce a mild nucleosome depletion on $A P 1$ promoter (Figure 4A). The effect on $A P 1$ intron is more pronounced. This region is closed in seedlings according to DNAseI signal and opens in the flower. Consistently, we observed a strong nucleosome depletion following LFY constitutive expression (Figure 4A). It is thus likely that LFY pioneer property is essential to trigger $A P 1$ activation through the opening of its closed intronic regulatory region. 
360 of animal pioneer TF (Zaret, 2020). Interestingly, LFY binding appears to be fully compatible 361 with the presence of histones, suggesting that LFY may require additional factors for histone displacement. The change in chromatin status following LFY binding might be due to LFY's capacity to recruit chromatin remodelers such as BRAHMA (BRM) and SPLAYED (SYD)

364 (Wu et al., 2012). These remodelers have been shown to have a very general role (Archacki et 365 al., 2016) and specific mutations altering LFY-BRM or LFY-SYD interactions are needed to fully test this hypothesis.

Our chromatin accessibility assay by FAIRE-qPCR suggests that constitutive LFY expression is sufficient to induce accessibility for a few key floral genes. To fully validate LFY pioneer activity, it would be essential to test its ability to alter chromatin states in the context of floral meristem cells, where the role of LFY is the most prominent and ideally using single cell isolation and next generation sequencing techniques. However, pioneer activity is likely a spectrum of activity in which TFs that play central roles in developmental transitions, such as LFY, are able to fulfill a pioneer role under certain chromatin conditions, in the presence of specific cofactors (Zaret, 2020) and/or for a few distinct loci (Li et al., 2019). Taken together, our in vitro and in vivo results demonstrate the essential properties of pioneer TFs- the competence to bind closed chromatin and the ability to trigger subsequent opening of these closed regions- are properties of LFY in the context of at least a few key floral regulatory targets. While this manuscript was in preparation, a preprint also describing LFY as a pioneer TF was made available on the bioRxiv server (Jin et al., 2020).

\section{Materials and methods}

\section{$381 \quad$ DAP-seq and ampDAP-seq}

\section{Plasmid construction}

383 Full-length LFY (AT5G61850.1, 420 residues) coding sequence was PCR amplified and cloned into pTnT vector (Promega) with an N-terminal 5XMyC tag to generate construct pTnT-5MyC385 LFY.

387 The input library of ampDAP-seq was PCR amplified from Col-0 genomic DNA (sheared to 388 average size of 200-500 bp by sonication) and constructed according to published protocol 389 (O'Malley et al., 2016; Bartlett et al., 2017; Lai et al., 2020). For input library of DAP-seq, the genomic DNA that retains native methylation pattern was extracted using phenol-chloroform 
391 from 2-weeks-old seedlings of $p 35 S:: L F Y$ line (pCA26 \#15) (Sayou et al., 2016) grown on 0.5x

392 Murashige and Skoog medium in long-day conditions.

393 DAP-seq and ampDAP-seq

394 LFY protein was produced using an in vitro transcription/translation system, TnT® SP6 High395 Yield Wheat Germ Protein Expression System (Promega L3260), according to the 396 manufacturer's instructions. In brief, $2 \mu \mathrm{g}$ input plasmid (pTnT-5MyC-LFY) was used in a 50 $397 \mu 1 \mathrm{TnT}$ reaction with $2-\mathrm{hr}$ incubation at $25^{\circ} \mathrm{C}$. DAP-seq was carried out according to published 398 protocol with minor modifications (O’Malley et al., 2016; Bartlett et al., 2017). Briefly, the 50 $399 \mu 1$ TnT reaction producing LFY was combined with $50 \mu 1$ IP buffer (20 mM Tris, pH7.5, 150 $400 \mathrm{mM} \mathrm{NaCl}, 1 \mathrm{mM}$ tris(2-carboxyethyl)phosphine, $0.005 \% \mathrm{NP}-40$, and proteinase inhibitor 401 (Roche)) and mixed with $20 \mu \mathrm{L}$ anti-MyC magnetic beads (Merck Millipore). Following $1 \mathrm{hr}$ 402 incubation at $4{ }^{\circ} \mathrm{C}$, the anti-MyC magnetic beads were immobilized and washed three times 403 with $100 \mu \mathrm{L}$ IP buffer. While the protein was still bound on anti-c-Myc magnetic beads, $50 \mathrm{ng}$ 404 DAP-seq or ampDAP-seq input library pre-ligated with Illumina adaptor sequences was added. 405 The DNA binding reaction was incubated at $4{ }^{\circ} \mathrm{C}$ on a rotor for 90 mins, and then washed 6 406 times using $100 \mu \mathrm{L}$ IP buffer. The bound DNA was heated to $98^{\circ} \mathrm{C}$ for $10 \mathrm{~min}$ and eluted in 30 407 $\mu 1$ EB buffer (10 mM Tris-Cl, pH 8.5). The eluted DNA fragments were PCR amplified using 408 Illumina TruSeq primers for 20 cycles, and purified using AMPure XP magnetic beads 409 (Beckman). The libraries were quantified by qPCR using NEBNext Library Quant Kit for 410 Illumina following manufacturer's instructions. Individual libraries were pooled with equal 411 molarity, and sequenced on Illumina HiSeq (Genewiz) with specification of paired-end 412 sequencing of 150 cycles. Each library obtained around 10 to 20 million raw reads. Both DAP413 seq and ampDAP-seq were performed in triplicates.

\section{Bioinformatic analyses}

415 Reads processing and peak calling

416 DAP-seq and ampDAP-seq read processing and peak calling was performed as previously 417 described (Lai et al., 2020). Briefly, reads were checked and cleaned using FastQC 418 (http://www.bioinformatics.babraham.ac.uk/projects/fastqc/), and NGmerge (Gaspar, 2018) 419 and mapped with bowtie2 (Langmead and Salzberg, 2012) onto the TAIR10 version of the $A$. 420 thaliana genome (www.arabidopsis.org), devoid of the mitochondrial and the chloroplast 421 genomes. The duplicated reads were removed using the samtools rmdup program (Li et al., 422 2009). The resulting alignment files for each sample were input to MACS2 (Zhang et al., 2008) to call peaks using the input DNA as control. Consensus peaks between replicates (when 
available) were defined using MSPC (Jalili et al., 2015) $\left(P\right.$-value cutoff $=10^{-4}$ ) for each experiment (DAP-seq, amplified DAP-seq and ChIP-seq from (Goslin et al., 2017)). Each consensus peak was scanned for possible subpeaks, split into several peaks if needed and the peak widths were then normalized to $\pm 200 \mathrm{bp}$ around the peak maximum. For all the resulting consensus peaks, a normalized coverage was computed as the mean of the normalized read coverage for each replicate (when replicates were available). Because the DAP-seq and ampDAP-seq experiment had different signal-to noise ratio (Supplemental Table 1) we used the total number of reads in consensus peaks for normalization. This normalized coverage (averaged across replica) defines the binding intensity of a TF at a bound region. The ratio of the binding intensity between DAP and ampDAP defines the DAP/ampDAP signal ratio. Measuring the effect of methylation on TF binding To measure the effect of cytosine methylation on the TF binding affinity, we tested the correlation between the DAP/ampDAP signal ratio and the methylation levels at 1) the whole bound regions, 2) at the TF best binding site (TFBS) in the bound region and 3) at each position of the TFBS. This approach markedly differs from previous analyses (O'Malley) where the change in binding affinity averaged across all binding sites was contrasted at methylated versus non-methylated regions in DAP and ampDAP experiments separately. By using the DAP/ampDAP signal ratio as a function of methylation levels at a same locus, our approach account for the variability across binding sites and better controls for differences in signal-tonoise ratio between ampDAP and DAP experiments. TFBSs were searched in bound regions using a position weight matrix (PWM) constructed for each TFs using MEME (Machanick and Bailey, 2011). The probability of cytosines methylation was taken from (Zhang et al., 2016). Methylation density (the number of methylated cytosines in a bound region) was defined as the number of cytosines with a probability of methylation greater than $50 \%$. Association between the relative binding intensity and methylation levels was assessed using Pearson's correlation tests from R package. The effect of methylation on LFY binding was compared to that of two others TFs (AT1G19210 and AT3G10030) for which DAP-seq and ampDAP-seq samples were available from (O’Malley et al., 2016).

453 The ChIP-seq datasets used were obtained from (Sayou et al., 2016) (1,954 peaks, two-week454 old seedlings 35S:LFY tissue), or re-computed (see above) from (Goslin et al., 2017) (884 peaks, inflorescence tissue of 35S:LFY-GR ap1 cal). Only the first 3,000 DAP-seq bound regions with lowest p-value were considered. Regions bound either in DAP-seq or in ChIP-seq were merged in a single bed file. When peaks overlapped for more than $80 \%$ of their respective 
458 length, they were considered as "common" and resized to create a common peak. Coverages of 459 pooled bound regions were retrieved for both datasets.

\section{DNA accessibility}

461 Closed and open chromatin regions were defined according to leaf DNaseI-seq dataset from 462 (Zhang et al., 2012). DNaseI-seq coverage was computed on the DAP and ChIP pooled peaks 463 and a peak was classified as open or closed following DHs regions from Zhang et al.

\section{Chromatin state}

4659 chromatin states was taken from (Sequeira-Mendes et al., 2014). Those chromatin states were 466 then crossed with our pooled peaks from ChIP-seq and DAP-seq and separated in deciles along 467 the ChIP-seq to DAP-seq coverage fold ratio (CFR).

\section{Nucleosomes}

469 MNase-seq defined genomic positions of nucleosomes in leaf were retrieved from (Zhang et 470 al., 2015). Custom python scripts were used to compute MNase-seq coverage for DNase471 defined closed and open bound regions. Peaks were then extended to $800 \mathrm{bp}$, around the 472 maximum, and sorted on their center ( $+/-100 \mathrm{bp}$ around the center).

\section{Position of LFYBS on nucleosomes}

474 LFY ChIP-seq bound regions were considered to be in an open chromatin state if they overlap 475 by more than $50 \%$ with a DNase peak in flower tissues, else they were considered to be in 476 closed chromatin. MNase data from flower tissues was crossed (using bedtools intersect $-\mathrm{f} 0.8$ 477 (Quinlan and Hall, 2010)) with ChIP-seq peaks to retain nucleosomes that are 80\% within a 478 LFY bound region. The resulting nucleosome sequences, plus half of a LFYBS (i.e. 9bp) at 479 both sides, were screened for LFYBS using a custom LFY position weight matrix (Sayou et al., 480 2016). We then counted the number of LFYBS, taking the center of the motif as reference, 481 present along the $147 \mathrm{bp}$ sequence of canonical nucleosome.

\section{Protein structural analysis}

483 The structure coordinates of LFY (accessions of 2vy1 and 2vy2 (Hamès et al., 2008)) are taken 484 from protein data bank (https://www.rcsb.org). The cytosine methylation mutation was done 485 using "Builder" option from the PyMOL GUI (www.pymol.org), all visualization was prepared 486 using PyMOL.

\section{Protein purification}

488 The protein AtLFY $\triangle 40$ was produced in E. coli Rosetta2 (DE3) strain (Novagen). Cells were 489 grown in Luria-Bertani medium supplemented with Kanamycin $(50 \mu \mathrm{g} / \mathrm{ml})$ and 
491 Betaine $(2 \mathrm{mM})$ was added and cultures were shifted to $18^{\circ} \mathrm{C}$ for $1 \mathrm{~h}$ before addition of $0.4 \mathrm{mM}$ 492 isopropyl $\beta$-D-1-thiogalactopyranoside. After overnight growth at $18^{\circ} \mathrm{C}$, cells were pelleted by 493 centrifugation. Pellets corresponding to 0.51 culture containing the recombinant protein were 494 sonicated in $50 \mathrm{ml}$ of lysis buffer (20 mM Tris- $\mathrm{HCl}$ pH 8.5, $1 \mathrm{mM}$ TCEP) supplemented by one 495 protease inhibitor cocktail tablet Complete EDTA-free (Roche) and centrifuged for $30 \mathrm{~min}$ at $49620,000 \mathrm{~g} 4^{\circ} \mathrm{C}$. The clear supernatant was transferred on a column containing $1 \mathrm{ml}$ Ni-Sepharose 497 High Performance resin (GE Healthcare), washed two times with lysis buffer containing 20 $498 \mathrm{mM}$ and $40 \mathrm{mM}$ imidazole, respectively, and eluted with lysis buffer containing $300 \mathrm{mM}$ 499 imidazole. Eluted fractions were immediately diluted three times in buffer without imidazole 500 and dialysed overnight. Protein concentrations were determined by SDS-PAGE, using a BSA 501 standard curve run on the same gel.

502 Recombinant histones were produced according to published protocols (Shim et al., 2012). The 503 coproduction of the Xenopus laevis four core histones was done using a pET29a polycistronic 504 plasmid containing the four core histones. The histoneH2A was tagged with N-terminal 505 hexahistidine (his6)-tag with a thrombin cleavage site. Histone H4 was tagged with a C506 terminal His6-tag preceded by a thrombin cleavage site. This plasmid was transformed in 507 BL21(DE3)pLysS bacteria. Cells were grown in Luria-Bertani medium supplemented with 508 Kanamycin $(50 \mu \mathrm{g} / \mathrm{ml})$ and Chloramphenicol $(34 \mu \mathrm{g} / \mathrm{ml})$ at $37^{\circ} \mathrm{C}$ under agitation up to an 509 optical density of $600 \mathrm{~nm}$ of 0.6 . were shifted to $18^{\circ} \mathrm{C}$ for $1 \mathrm{~h}$ before addition of $0.4 \mathrm{mM}$ 510 isopropyl $\beta$-D-1-thiogalactopyranoside. cells were pelleted by centrifugation. Pellets 511 containing the recombinant protein were sonicated in $50 \mathrm{ml}$ of lysis buffer $(20 \mathrm{mM}$ Tris- $\mathrm{HCl}$ $512 \mathrm{pH} 7.5,2 \mathrm{M} \mathrm{NaCl} 1 \mathrm{mM}$ TCEP) supplemented by one protease inhibitor cocktail tablet 513 Complete EDTA-free (Roche) and centrifuged for $30 \mathrm{~min}$ at $18,000 \mathrm{~g} 4^{\circ} \mathrm{C}$. The clear 514 supernatant was transferred on a column containing $3 \mathrm{ml} \mathrm{Ni-Sepharose} \mathrm{High} \mathrm{Performance} \mathrm{resin}$ 515 (GE Healthcare), washed two times with lysis buffer containing $30 \mathrm{mM}$ and $50 \mathrm{mM}$ imidazole, 516 respectively. Elution was performed with lysis buffer containing $300 \mathrm{mM}$ imidazole. Fraction 517 were analyzed on $18 \%$ SDS-PAGE. Thrombin digestion was carried out by adding purified 518 thrombin in 25:1 mass ratio and incubating the samples at room temperature for 4 hours. The 519 digestion was confirmed by SDS-PAGE. Digested histones were then concentrated by centrifugation using amicon membrane (MW50KDa) at $4^{\circ} \mathrm{C}$ et $4,000 \mathrm{xg}$ during $20 \mathrm{~min}$ intervals, with gentle mixing between each centrifugation. The concentrated sample was then injected onto a Superdex 200 10/300 column in lysis buffer. The histone octamer peak was eluted at an elution volume of $12.8 \mathrm{ml}$. the peaks fractions were pooled and concentrated at a concentration of $1.84 \mathrm{mg} / \mathrm{ml}$ and aliquoted flash-frozen in the presence of $50 \%$ glycerol. 
526 DNA sequences used for nucleosome reconstruction

527 To test the interaction between AtLFY $\triangle 40$ and nucleosomes we reconstituted nucleosomes 528 with the 601 sequences and different 601 sequences containing a LFY binding site at different 529 positions (C1-C7 and E1-E7, Figure 3B). As a control, we also tested RAX1 nucleosomal DNA 530 binding using 601 sequence containing a RAX1 binding site (C1-C7, Figure 3B). All cloned in 531 a pUC57 plasmid (sequences see Supplemental Table 2 and 3). Sequences of an $A P 1$ intronic 532 region and $A P 1$ promoter region (Figure 3D) were also cloned in pUC57 and used for 533 nucleosomal reconstitution (sequences see Supplemental Table 2).

534 All fragments used for nucleosome reconstruction were amplified by PCR using Invitrogen 535 primer labelled with CY5 fluorophore. The resulting fragment was then checked by $1 \%$ agarose 536 gel and purified with the Monarch ${ }^{\circledR}$ DNA Gel Extraction Kit (New England Biolabs). The 537 purified fragment was precipitated by ethanol precipitation and resuspended in buffer $(25 \mathrm{mM}$ 538 Tris, $\mathrm{pH} 7.5,2 \mathrm{M} \mathrm{NaCl}$ and $1 \mathrm{mM} \mathrm{TCEP}$ ) and adjusted at a concentration of $200 \mathrm{ng} / \mu \mathrm{l}$.

\section{Nucleosome reconstruction}

540 The nucleosomes assembly was performed by salt dilution method (Okuwaki et al., 2005). 541 Briefly, DNA of interest and recombinant histone octamer were mixed at a molar ratio (DNA / 542 histones) between $1 / 1$ and $1 / 1.2$ in a solution of $25 \mathrm{mM}$ Tris $\mathrm{pH} 7.52 \mathrm{M} \mathrm{NaCl} 1 \mathrm{mM}$ TCEP. This $543 \mathrm{mix}$ was incubated at $30^{\circ} \mathrm{C}$ for $20 \mathrm{~min}$. The reaction was serially diluted to $1.5,1,0.8,0.6,0.5$, $5440.4,0.3$, and $0.2 \mathrm{M} \mathrm{NaCl}$ using buffer $25 \mathrm{mM}$ Tris $\mathrm{pH} 7.51 \mathrm{mM}$ TCEP with 20 min incubation 545 at $30^{\circ} \mathrm{C}$ for each dilution step. The reconstitution was confirmed by native gel analysis.

\section{Electrophoretic Mobility Shift Assay}

547 Nucleosomes of interest were incubated with $500 \mu \mathrm{M}$ AtLFY $\Delta 40$ in buffer $(25 \mathrm{~mm}$ Tris pH7.5 $548200 \mathrm{mM} \mathrm{NaCl} 1 \mathrm{mM}$ TCEP $10 \%$ glycerol $0.1 \mathrm{mg} / \mathrm{ml}$ BSA $0,12 \mathrm{mg} / \mathrm{ml}$ herring and salmon sperm 549 DNA for 1 hour at room temperature. The different complex was separated on 5\% non550 denaturing polyacrylamide gels run in $0.5 \mathrm{X}$ Tris-borate-EDTA (TBE) buffer. Gels run for one 551 hour at $4^{\circ} \mathrm{C}$ at $120 \mathrm{~V}$. Complexes were visualized with Cy5 - exposition filter (ChemiDoc MP 552 Imager; BIO-RAD).

\section{Formaldehyde-Assisted Isolation of Regulatory Elements (FAIRE)-qPCR}

554 Site selection for FAIRE-qPCR

555 To test the effect of LFY binding on nucleosomes, LFY ChIP-seq peaks (Sayou et al., 2016) 556 with a nucleosome in leaf tissue (overlap $>=50 \%$ ) and no nucleosomes in floral tissue were 
557

558

559

560

561

562

563

564

565

566

567

568

569

570

571

572

573

574

575

576

577

578

579

580

581

582

583

584

585

586

587

selected (Zhang et al., 2015). Selected peaks were then attributed to nearby genes (peaks within $3 \mathrm{~kb}$ upstream and $1 \mathrm{~kb}$ downstream; overlap $>=80 \%$ ). In those regions, we selected two known LFY targets, APl (AT1G69120) and $A G$ (AT4G18960), and ULT1 (AT4G28190), another floral regulator. We applied a similar method to select three control regions that are not bound by LFY and are occupied with nucleosomes in leaf and floral tissue (AT3G18780 (Actin2), AT2G38220 and AT4G22285).

\section{FAIRE-qPCR}

FAIRE assays were performed on two-week-old seedlings of Arabidopsis thaliana ecotype Columbia (Col-0) background and 35S::LFY. Seeds were surface-sterilized by treatment with bayrochlore, washed, then sown in sterile half-strength MS medium and placed for 2-4 days at $4{ }^{\circ} \mathrm{C}$ to obtain homogeneous germination. Plants were grown in square petri dishes in growth chambers at $20{ }^{\circ} \mathrm{C}$ under long-day (16 h of light) conditions. $1 \mathrm{~g}$ of plant material was then crosslinked in $1 \%(\mathrm{v} / \mathrm{v})$ formaldehyde at room temperature for $15 \mathrm{~min}$ using vacuum infiltration. Crosslinking was quenched by adding glycine solution to a final concentration of $0.125 \mathrm{M}$ under vacuum infiltration for 5 minutes. The crosslinked plantlets were ground into powder using liquid nitrogen and nuclei were isolated using Nuclei Isolation Buffer $(0,25 \mathrm{M}$ Sucrose, 10mM Tris-HCl pH8, 10mM MgCl2, 1\% Triton X-100, 5mM $\beta$-mercaptoethanol, and proteases inhibitors) and then resuspended in $1 \mathrm{ml}$ of FAIRE Lysis Buffer (0,1\% SDS, $50 \mathrm{~mm}$ Tris- $\mathrm{HCl}$ pH 8, 10 mm ethylene diamine tetraacetic acid (EDTA) pH 8). The crosslinked DNA was sheared to an average size of 200 - 300 bp using a Covaris S220 (Peak Power: 175W, cycles/burst: 200, Duty Factory: 20, 4min). Samples were centrifuged for 15 min and 13,000 x $\mathrm{g}$ at $4{ }^{\circ} \mathrm{C}$ and the supernatant was transferred into new tubes. A $100 \mu 1$ aliquot was used as control DNA and directly treated with $1 \mu \mathrm{l}$ of RNAse A+T1 cocktail enzyme mix (Thermo Fisher Scientific) for $1 \mathrm{~h}$ at $37^{\circ} \mathrm{C}$ followed by proteinase $\mathrm{K}$ treatment for $4 \mathrm{~h}$ at $37^{\circ} \mathrm{C}$ and $6 \mathrm{~h}$ at $65^{\circ} \mathrm{C}$ to reverse the crosslinks. The non-de-crosslinked samples were RNAse A+T1 treated as for control DNA and a phenol:chloroform:isoamyl alcohol (25:24:1) extraction was performed to purify DNA and control DNA in a final volume of $100 \mu \mathrm{L}$ TE buffer $(10 \mathrm{mM}$ Tris $\mathrm{pH} 8$, $1 \mathrm{mM}$ EDTA). The non-de-crosslinked free DNA samples were then incubated overnight at 65 ${ }^{\circ} \mathrm{C}$ to remove inter-DNA crosslinks. DNA was quantified using Qubit ds DNA HS kit (Thermo Fisher Scientific) and the ratio between nucleosome-free DNA versus total DNA was determined by qPCR analysis using 20ng of template DNA for each reaction.

\section{Accession Numbers}


LFY DAP-seq sequencing data from this article can be found in the NCBI GEO data libraries under accession numbers GSE160013 (token afwnayckrrajfev for reviewers).

\section{Acknowledgements}

We thank K. Kaufmann for discussions. This project was supported by the ANR-DFG project Flopinet (ANR-16-CE92-0023-01) to CZ and FP, the GRAL LabEX (ANR-10-LABX-49-01) with the frame of the CBH-EUR-GS (ANR-17-EURE-0003) to CZ, FP and AS.

\section{Authors contributions}

FP, CZ, RD designed and supervised the project, RBM, JL, AS performed bioinformatics analyses, LG, GV, JLM, HD, ET and EBH performed biochemical analyses, XL performed DAP-seq, YW performed FAIRE supervised by MB and DL, FP, CZ and XL wrote the paper with the help of all authors.

\section{References}

Archacki, R., Yatusevich, R., Buszewicz, D., Krzyczmonik, K., Patryn, J., IwanickaNowicka, R., Biecek, P., Wilczynski, B., Koblowska, M., Jerzmanowski, A., et al. (2017). Arabidopsis SWI/SNF chromatin remodeling complex binds both promoters and terminators to regulate gene expression. Nuc. Acids Res. 45(6):3116-3129.

Bartlett, A., O'Malley, R. C., Huang, S. C., Galli, M., Nery, J. R., Gallavotti, A., and Ecker, J. R. (2017). Mapping genome-wide transcription-factor binding sites using DAP-seq. Nat. Protocols 12:1659-1672.

Benlloch, R., Kim, M. C., Sayou, C., Thévenon, E., Parcy, F., and Nilsson, O. (2011). Integrating long-day flowering signals: A LEAFY binding site is essential for proper photoperiodic activation of APETALA1. Plant J. 67:1094-1102.

Calonje, M., Sanchez, R., Chen, L., and Sung, Z. R. (2008). EMBRYONIC FLOWER1 Participates in Polycomb Group-Mediated AG Gene Silencing in Arabidopsis. Plant Cell 20:277291.

Chae, E., Tan, Q. K.-G., Hill, T. A., and Irish, V. F. (2008). An Arabidopsis F-box protein acts as a transcriptional co-factor to regulate floral development. Development 135:12351245.

Chahtane, H., Vachon, G., Le Masson, M., Thévenon, E., Périgon, S., Mihajlovic, N., Kalinina, A., Michard, R., Moyroud, E., Monniaux, M., et al. (2013). A variant of LEAFY reveals its capacity to stimulate meristem development by inducing RAX1. Plant J. 74:678689. 
Chodavarapu, R. K., Feng, S., Bernatavichute, Y. V., Chen, P.-Y., Stroud, H., Yu, Y., Hetzel, J. A., Kuo, F., Kim, J., Cokus, S. J., et al. (2010). Relationship between nucleosome positioning and DNA methylation. Nature 466:388-392.

Chua, E. Y. D., Vasudevan, D., Davey, G. E., Wu, B., and Davey, C. A. (2012). The mechanics behind DNA sequence-dependent properties of the nucleosome. Nuc. Acids Res. 40:6338-6352.

Denay, G., Chahtane, H., Tichtinsky, G., and Parcy, F. (2017). A flower is born: an update on Arabidopsis floral meristem formation. Cur. Op. Plant Biol. 35:15-22.

Dodonova, S. O., Zhu, F., Dienemann, C., Taipale, J., and Cramer, P. (2020). Nucleosomebound SOX2 and SOX11 structures elucidate pioneer factor function. Nature . 580, $669-672$

Fernandez Garcia, M., Moore, C. D., Schulz, K. N., Alberto, O., Donague, G., Harrison, M. M., Zhu, H., and Zaret, K. S. (2019). Structural Features of Transcription Factors Associating with Nucleosome Binding. Mol. Cell 75, 5, 921-932.

Gallois, J.-L., Nora, F. R., Mizukami, Y., and Sablowski, R. (2004). WUSCHEL induces shoot stem cell activity and developmental plasticity in the root meristem. Genes \& Dev. 18:375-380.

Gaspar, J. M. (2018). NGmerge: merging paired-end reads via novel empirically-derived models of sequencing errors. BMC Bioinformatics 19:536.

Goodrich, J., Puangsomlee, P., Martin, M., Long, D., Meyerowitz, E. M., and Coupland, G. (1997). A Polycomb-group gene regulates homeotic gene expression in Arabidopsis. Nature 386:44-51.

Goslin, K., Zheng, B., Serrano-Mislata, A., Rae, L., Ryan, P. T., Kwaśniewska, K., Thomson, B., Ó'Maoiléidigh, D. S., Madueño, F., Wellmer, F., et al. (2017). Transcription Factor Interplay between LEAFY and APETALA1/CAULIFLOWER during Floral Initiation. Plant Physiol. 174:1097-1109.

Hamès, C., Ptchelkine, D., Grimm, C., Thevenon, E., Moyroud, E., Gérard, F., Martiel, J.-L., Benlloch, R., Parcy, F., and Müller, C. W. (2008). Structural basis for LEAFY floral switch function and similarity with helix-turn-helix proteins. EMBO J. 27:2628-2637.

Hu, G., Schones, D. E., Cui, K., Ybarra, R., Northrup, D., Tang, Q., Gattinoni, L., Restifo, N. P., Huang, S., and Zhao, K. (2011). Regulation of nucleosome landscape and transcription factor targeting at tissue-specific enhancers by BRG1. Genome Res. 21:1650-1658.

Hu, S., Wan, J., Su, Y., Song, Q., Zeng, Y., Nguyen, H. N., Shin, J., Cox, E., Rho, H. S., Woodard, C., et al. (2013). DNA methylation presents distinct binding sites for human transcription factors. elife 2:e00726. 
Iwafuchi-Doi, M. (2018). The mechanistic basis for chromatin regulation by pioneer transcription factors. Wiley Interdisciplinary Reviews: Systems Biology and Medicine 11(1):e1427.

Iwafuchi-Doi, M., and Zaret, K. S. (2014). Pioneer transcription factors in cell reprogramming. Genes Dev. 28:989-998.

Iwafuchi-Doi, M., and Zaret, K. S. (2016). Cell fate control by pioneer transcription factors. Development 143:1833-1837.

Jalili, V., Matteucci, M., Masseroli, M., and Morelli, M. J. (2015). Using combined evidence from replicates to evaluate ChIP-seq peaks. Bioinformatics 31:2761-2769.

Jin, R., Klasfeld, S., Garcia, M. F., Xiao, J., Han, S.-K., Konkol, A., Zhu, Y., and Wagner, D. (2020). LEAFY is a pioneer transcription factor and licenses cell reprogramming to floral fate. BioRxiv Advance Access published March 18, 2020, doi:10.1101/2020.03.16.994418.

Kaufmann, K., Pajoro, A., and Angenent, G. C. (2010). Regulation of transcription in plants: mechanisms controlling developmental switches. Nat. Rev. Genet. 11:830-842.

King, H. W., and Klose, R. J. (2017). The pioneer factor OCT4 requires the chromatin remodeller BRG1 to support gene regulatory element function in mouse embryonic stem cells. elife 6:1-24.

Klemm, S. L., Shipony, Z., and Greenleaf, W. J. (2019). Chromatin accessibility and the regulatory epigenome. Nat. Rev. Genet. 20:207-220.

Lai, X., Stigliani, A., Lucas, J., Hugouvieux, V., Parcy, F., and Zubieta, C. (2020). Genome-wide binding of SEPALLATA3 and AGAMOUS complexes determined by sequential DNAaffinity purification sequencing. Nuc. Acids Res. 48:9637-9648.

Langmead, B., and Salzberg, S. L. (2012). Fast gapped-read alignment with Bowtie 2. Nature Methods 9:357-359.

Levin, J.Z. and Meyerowitz, E. M. (1995). UFO: An Arabidopsis Gene Involved in Both Floral Meristem and Floral Organ Development. Plant Cell 7:529-548.

Li, H., Handsaker, B., Wysoker, A., Fennell, T., Ruan, J., Homer, N., Marth, G., Abecasis, G., Durbin, R., and 1000 Genome Project Data Processing Subgroup (2009). The Sequence Alignment/Map format and SAMtools. Bioinformatics 25:2078-2079.

Li, S., Bo Zheng, E., Zhao, L., and Liu, S. (2019). Nonreciprocal and Conditional Cooperativity Directs the Pioneer Activity of Pluripotency Transcription Factors. Cell Reports 28, 10 : 2689-2703.e4

Liu, Y., Olanrewaju, Y. O., Zheng, Y., Hashimoto, H., Blumenthal, R. M., Zhang, X., and Cheng, X. (2014). Structural basis for Klf4 recognition of methylated DNA. Nuc. Acids Res. 42:4859-4867. 
Lohmann, J. U., Hong, R. L., Hobe, M., Busch, M. A., Parcy, F., Simon, R., and Weigel, D. (2001). A Molecular Link between Stem Cell Regulation and Floral Patterning in Arabidopsis. Cell 105:793-803.

Lowary, P. T., and Widom, J. (1998). New DNA sequence rules for high affinity binding to histone octamer and sequence-directed nucleosome positioning. J. Mol. Biol. 276:19-42.

Machanick, P., and Bailey, T. L. (2011). MEME-ChIP: Motif analysis of large DNA datasets. Bioinformatics 27:1696-1697.

Magnani, L., Eeckhoute, J., and Lupien, M. (2011). Pioneer factors: Directing transcriptional regulators within the chromatin environment. Trends Genet. 27:465-474.

Mayran, A., and Drouin, J. (2018). Pioneer transcription factors shape the epigenetic landscape. J. Biol. Chem. 7293(36):13795-13804.

Mayran, A., Khetchoumian, K., Hariri, F., Pastinen, T., Gauthier, Y., Balsalobre, A., and Drouin, J. (2018). Pioneer factor Pax7 deploys a stable enhancer repertoire for specification of cell fate. Nat. Genet. 50: 259-269.

McGinty, R. K., and Tan, S. (2015). Nucleosome Structure and Function. Chem. Rev. 115:2255-2273.

Michael, A. K., Grand, R. S., Isbel, L., Cavadini, S., Kozicka, Z., Kempf, G., Bunker, R. D., Schenk, A. D., Graff-Meyer, A., Pathare, G. R., et al. (2020). Mechanisms of OCT4SOX2 motif readout on nucleosomes. Science 368, 6498:1460-1465.

Moreau, F., Thévenon, E., Blanvillain, R., Lopez-Vidriero, I., Franco-Zorrilla, J. M., Dumas, R., Parcy, F., Morel, P., Trehin, C., and Carles, C. C. (2016). The Myb-domain protein ULTRAPETALA1 INTERACTING FACTOR 1 controls floral meristem activities in Arabidopsis. Development 143:1108-1119.

Moyroud, E., Kusters, E., Monniaux, M., Koes, R., and Parcy, F. (2010). LEAFY blossoms. Trends Plant Sci. 15:346-352.

Moyroud, E., Minguet, E. G., Ott, F., Yant, L., Posé, D., Monniaux, M., Blanchet, S., Bastien, et al. (2011). Prediction of regulatory interactions from genome sequences using a biophysical model for the Arabidopsis LEAFY transcription factor. Plant Cell 23:12931306.

Okuwaki, M., Kato, K., Shimahara, H., Tate, S., and Nagata, K. (2005). Assembly and Disassembly of Nucleosome Core Particles Containing Histone Variants by Human Nucleosome Assembly Protein I. MCB 25:10639-10651.

O’Malley, R. C., Huang, S. shan C., Song, L., Lewsey, M. G., Bartlett, A., Nery, J. R., Galli, M., Gallavotti, A., and Ecker, J. R. (2016). Cistrome and Epicistrome Features Shape the Regulatory DNA Landscape. Cell 166:1598. 
Pajoro, A., Madrigal, P., Muiño, J. M., Matus, J. T., Jin, J., Mecchia, M. A., Debernardi, J. M., Palatnik, J. F., Balazadeh, S., Arif, M., et al. (2014). Dynamics of chromatin accessibility and gene regulation by MADS-domain transcription factors in flower development. Genome Biol. 15:R41.

Parcy, F., Nilsson, O., Busch, M. A., Lee, I., and Weigel, D. (1998). A genetic framework for floral patterning. Nature 395:561-566.

Quinlan, A. R., and Hall, I. M. (2010). BEDTools: a flexible suite of utilities for comparing genomic features. Bioinformatics 26:841-842.

Risseeuw, E., Venglat, P., Xiang, D., Komendant, K., Daskalchuk, T., Babic, V., Crosby, W., and Datla, R. (2013). An activated form of UFO alters leaf development and produces ectopic floral and inflorescence meristems. PLOS ONE 8.

Sayou, C., Nanao, M. H., Jamin, M., Pose, D., Thevenon, E., Gregoire, L., Tichtinsky, G., Denay, G., Ott, F., Llobet, M. P., et al. (2016). A SAM oligomerization domain shapes the genomic binding landscape of the LEAFY transcription factor. Nat. Commun. 7:11222.

Sequeira-Mendes, J., Araguez, I., Peiro, R., Mendez-Giraldez, R., Zhang, X., Jacobsen, S. E., Bastolla, U., and Gutierrez, C. (2014). The Functional Topography of the Arabidopsis Genome Is Organized in a Reduced Number of Linear Motifs of Chromatin States. Plant Cell 26:2351-2366.

Sherwood, R. I., Hashimoto, T., O'Donnell, C. W., Lewis, S., Barkal, A. A., Van Hoff, J. P., Karun, V., Jaakkola, T., and Gifford, D. K. (2014). Discovery of directional and nondirectional pioneer transcription factors by modeling DNase profile magnitude and shape. Nat. Biotech. 32:171-178.

Shim, Y., Duan, M.-R., Chen, X., Smerdon, M. J., and Min, J.-H. (2012). Polycistronic coexpression and nondenaturing purification of histone octamers. Ana. Biochem. 427:190-192.

Slattery, M., Zhou, T., Yang, L., Dantas Machado, A. C., Gordân, R., and Rohs, R. (2014). Absence of a simple code: How transcription factors read the genome. Trends Biochem. Sci. 39:381-399.

Soufi, A., Garcia, M. F., Jaroszewicz, A., Osman, N., Pellegrini, M., and Zaret, K. S. (2015). Pioneer transcription factors target partial DNA motifs on nucleosomes to initiate reprogramming. Cell 161:555-568.

Tao, Z., Shen, L., Gu, X., Wang, Y., Yu, H., and He, Y. (2017). Embryonic epigenetic reprogramming by a pioneer transcription factor in plants. Nature 551:124-128.

Turck, F., Roudier, F., Farrona, Sara., Martin-Magniette, M.-L., Guillaume, E., Buisine, N., Gagnot, S., Martienssen, R. A., Coupland, G., and Colot, V. (2007). Arabidopsis TFL2/LHP1 Specifically Associates with Genes Marked by Trimethylation of Histone H3 Lysine 27. PLoS Genetics 3(6):e86. 
Wagner, D. (1999). Transcriptional Activation of APETALA1 by LEAFY. Science 285:582-584.

Weber, E., Buzovetsky, O., Heston, L., Yu, K.-P., Knecht, K. M., El-Guindy, A., Miller, G., and Xiong, Y. (2019). A Noncanonical Basic Motif of Epstein-Barr Virus ZEBRA Protein Facilitates Recognition of Methylated DNA, High-Affinity DNA Binding, and Lytic Activation. J Virol 93:e00724-19.

Winter, C. M., Austin, R. S., Blanvillain-Baufumé, S., Reback, M. A., Monniaux, M., Wu, M. F., Sang, Y., Yamaguchi, A., Yamaguchi, N., Parker, J. E., et al. (2011). LEAFY Target Genes Reveal Floral Regulatory Logic, cis Motifs, and a Link to Biotic Stimulus Response. Dev. Cell 20:430-443.

Wu, M., Sang, Y., Bezhani, S., Yamaguchi, N., Han, S., Li, Z., Su, Y., Slewinski, T. L., and Wagner, D. (2012). SWI2/SNF2 chromatin remodeling ATPases overcome polycomb repression and control floral organ identity with the LEAFY and SEPALLATA3 transcription factors. Proc. Nat. Acad. Sci.U S A 109:3576-3581.

Yamaguchi, N., Wu, M. F., Winter, C. M., Berns, M. C., Nole-Wilson, S., Yamaguchi, A., Coupland, G., Krizek, B. A., and Wagner, D. (2013). A Molecular Framework for AuxinMediated Initiation of Flower Primordia. Dev. Cell 24:271-282.

Yin, Y., Morgunova, E., Jolma, A., Kaasinen, E., Sahu, B., Khund-Sayeed, S., Das, P. K., Kivioja, T., Dave, K., Zhong, F., et al. (2017). Impact of cytosine methylation on DNA binding specificities of human transcription factors. Science 356:eaaj2239.

Zaret, K. S. (2020). Pioneer Transcription Factors Initiating Gene Network Changes. Annu. Rev. Genet. 54:annurev-genet-030220-015007.

Zhang, Y., Liu, T., Meyer, C. A., Eeckhoute, J., Johnson, D. S., Bernstein, B. E., Nussbaum, C., Myers, R. M., Brown, M., Li, W., et al. (2008). Model-based Analysis of ChIP-Seq (MACS). Genome Biol. 9:R137.

Zhang, W., Zhang, T., Wu, Y., and Jiang, J. (2012). Genome-Wide Identification of Regulatory DNA Elements and Protein-Binding Footprints Using Signatures of Open Chromatin in Arabidopsis. Plant Cell 24:2719-2731.

Zhang, T., Zhang, W., and Jiang, J. (2015). Genome-Wide Nucleosome Occupancy and Positioning and Their Impact on Gene Expression and Evolution in Plants. Plant Physiol. 168:1406-1416.

Zhang, Q., Wang, D., Lang, Z., He, L., Yang, L., Zeng, L., Li, Y., Zhao, C., Huang, H., Zhang, H., et al. (2016). Methylation interactions in Arabidopsis hybrids require RNA-directed DNA methylation and are influenced by genetic variation. Proc. Natl. Acad. Sci. USA 113:E4248-E4256.

Zhu, H., Wang, G., and Qian, J. (2016). Transcription factors as readers and effectors of DNA methylation. Nat. Rev. Genet. 17:551-565. 


\section{Supplementary information}

809

812 Xuelei Lai ${ }^{1, *}$, Romain Blanc-Mathieu ${ }^{1, *}$, Loïc GrandVuillemin ${ }^{1, \wedge}$, Ying Huang ${ }^{2, \wedge}$, Arnaud

813 Stigliani ${ }^{1,3}$, Jérémy Lucas ${ }^{1}$, Emmanuel Thévenon ${ }^{1}$, Jeanne Loue-Manifel ${ }^{1,4}$, Hussein Daher ${ }^{1,5}$,

814 Eugenia Brun-Hernandez ${ }^{1}$, Gilles Vachon ${ }^{1}$, David Latrasse ${ }^{2}$, Moussa Benhamed ${ }^{2}$, Renaud

815 Dumas $^{1}$, Chloe Zubieta ${ }^{1}$ and Francois Parcy ${ }^{1}$

816

817

818 This file contains:

81911 supplementary figures

8203 supplementary tables.

821

822 The first 6 figures are related to Figure 1, Figure S7 relates to Figure 2, Figure S8 to Figure 3, 823 and Figures S9-S11 to Figure 4 
826
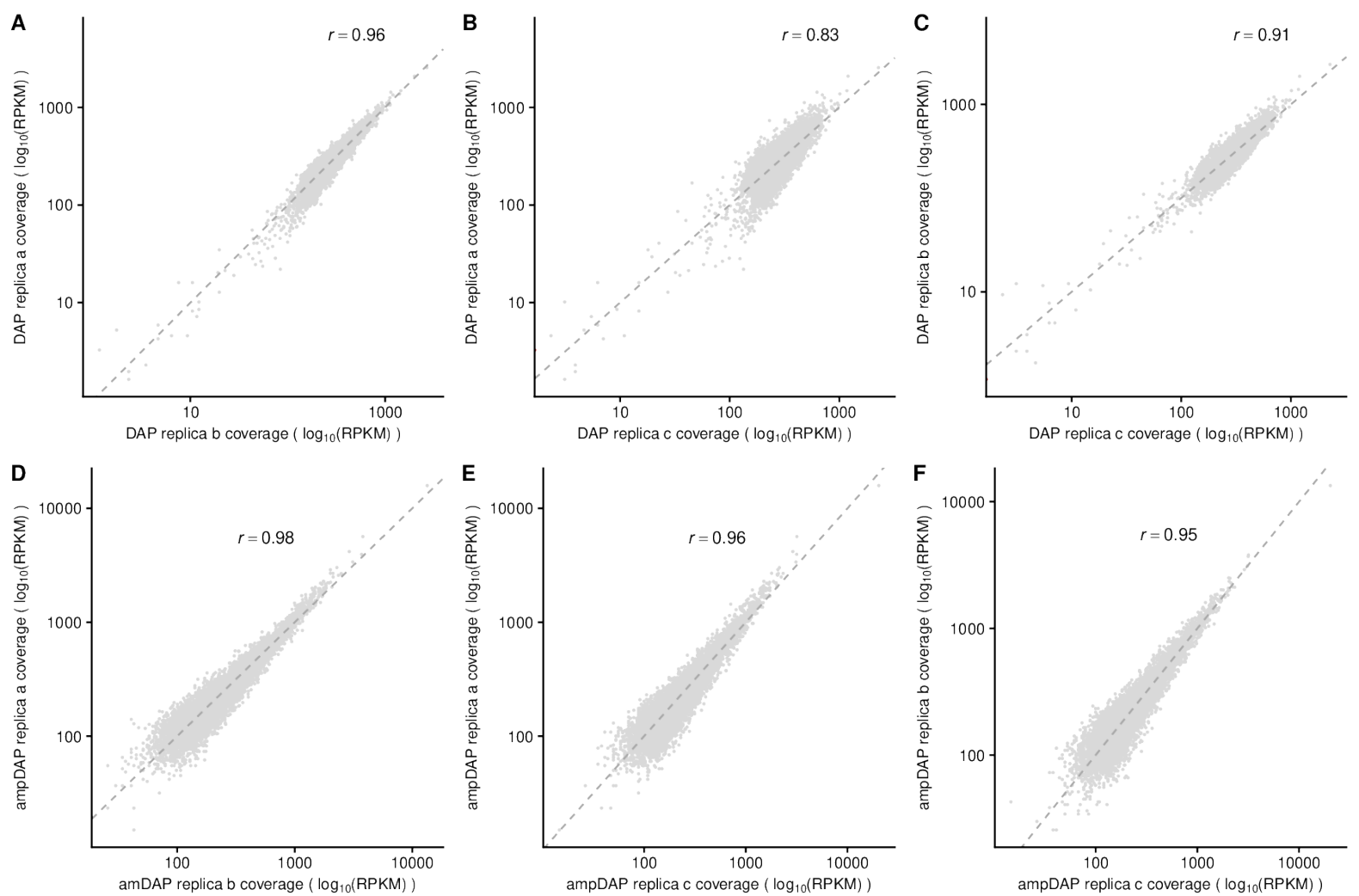

829 Supplementary Figure 1: Replicate reproducibility of LFY DAP-seq experiments.

830 Scatter plots of coverage, normalized by the total number of reads in peaks, between three

831 replicates (a,b,c) of DAP-seq (first row) and amplified DAP-seq (second row) experiments, with 832 Pearson's correlation at top. 
A

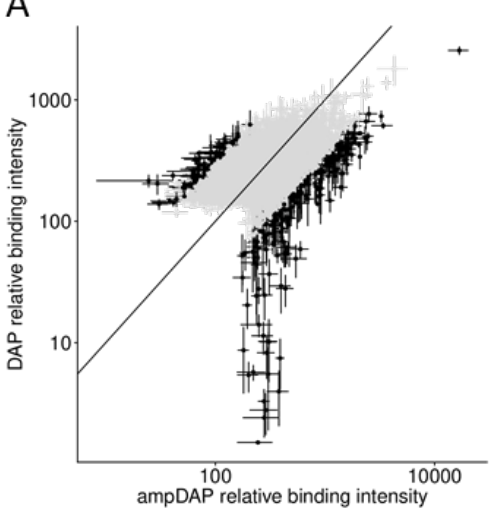

B

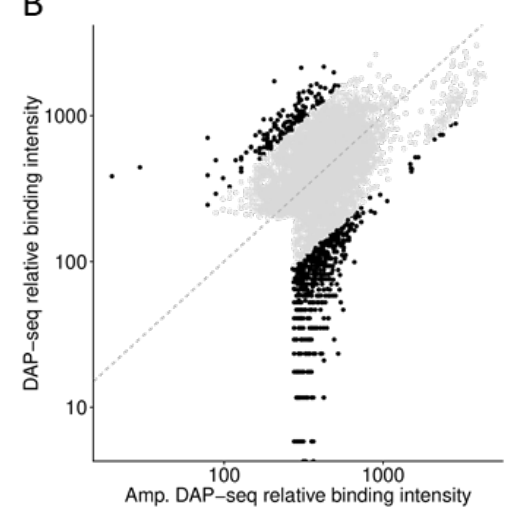

C

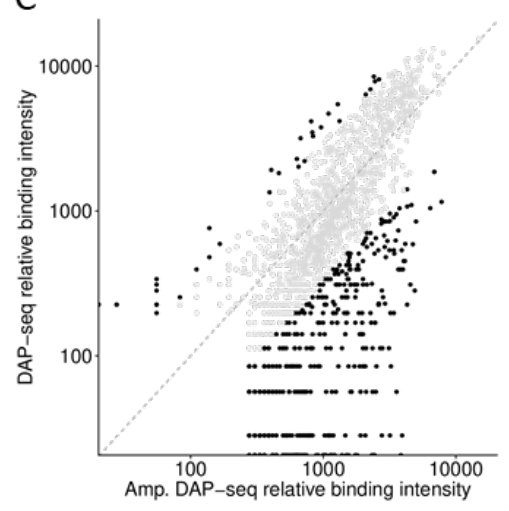

837 Supplementary Figure 2: Relative binding intensity for ampDAP-seq and DAP-seq

838 experiments for LFY (A), ERF07(AT1G19210) (B), and a trihelix-domain containing protein

839 (AT3G10030) (C).

840 The relative binding intensity is expressed as the number of reads in the bound region

841 normalized by its length and by the total number of reads in all bound regions. Black and grey

842 separate bound regions with a difference in relative binding intensity of ampDAP versus DAP-

843 seq experiment greater and less than 3-fold. Variability across replicates (available for LFY

844 only) is represented by error bars. 
846 A
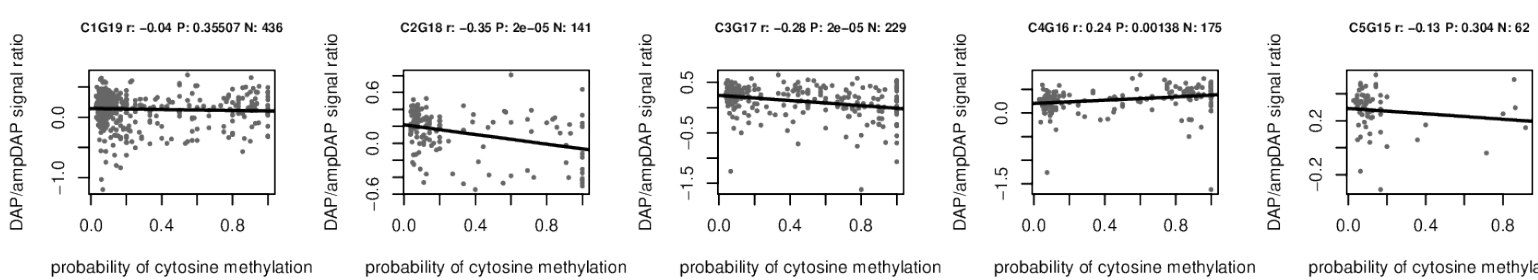

probability of cytosine methylatio

probability of cytosine methylation

probability of cytosine methylation

probability of cytosine methylatio

probability of cytosine methylation
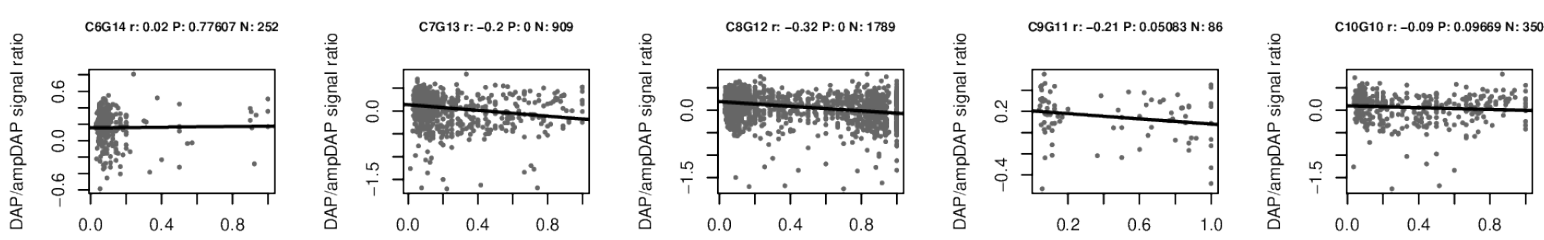

847

probability of cytosine methylation

probability of cytosine methylation

probability of cytosine methylation

probability of cytosine methylation

probability of cytosine methylation

848 в
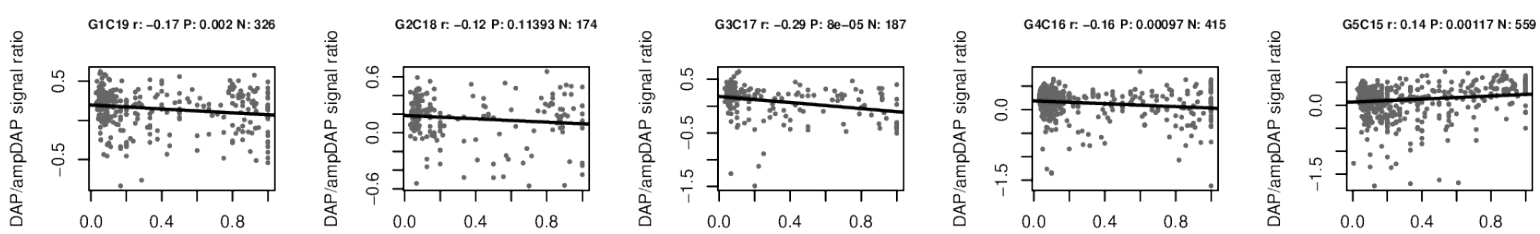

probability of cytosine methylation

probability of cytosine methylation

probability of cytosine methylation

probability of cytosine methylation

probability of cytosine methylation
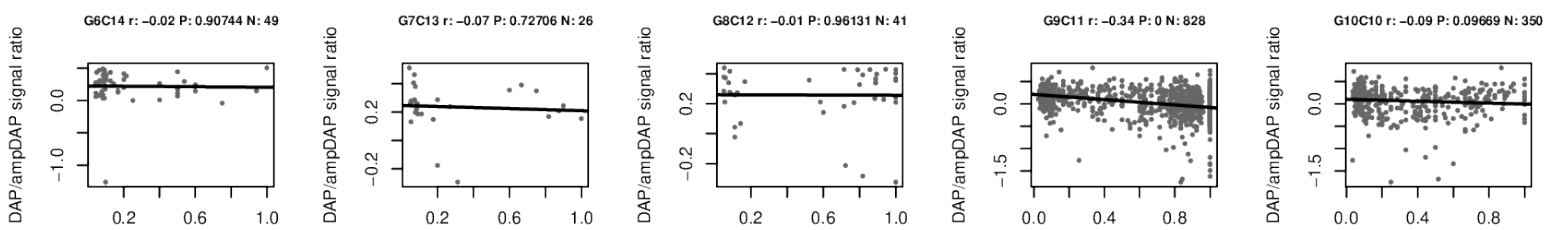

851 Supplementary Figure 3: Effect of methylation of each individual position on LFY binding.

852 Relation between methylation probability at a single site in the predicted best LFY binding

853 site and the $\log _{10}$-scaled relative binding site intensity of a DAP-seq versus an ampDAP-seq

854 experiment for LFY on the forward (A) and reverse (B) strand. 


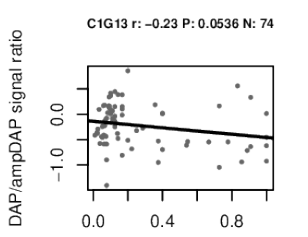

probability of cytosine methylation

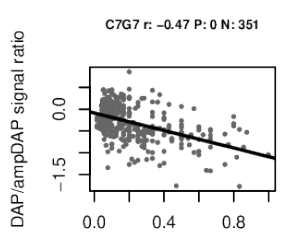

858

859

probability of cytosine methylation

B

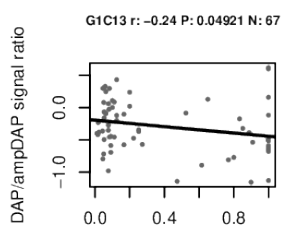

probability of cytosine methylation

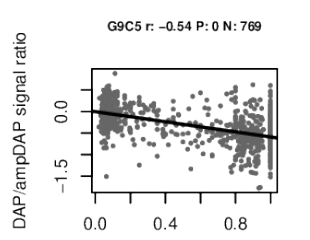

probability of cytosine methylation

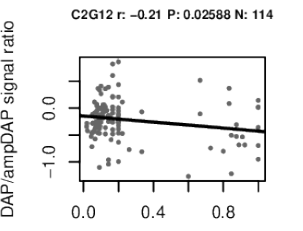

probability of cytosine methylation

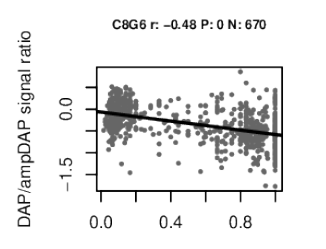

probability of cytosine methylation

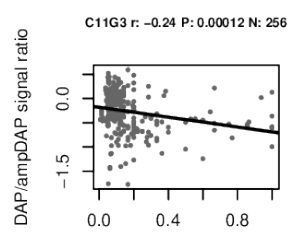

probability of cytosine methylation

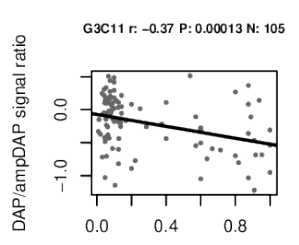

probability of cytosine methylation

probability of cytosine methylation

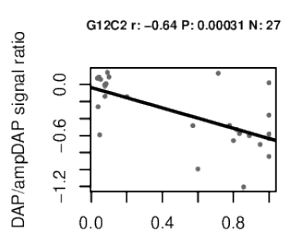

probability of cytosine methylation

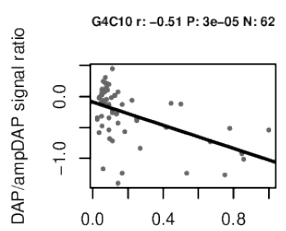

probability of cytosine methylation
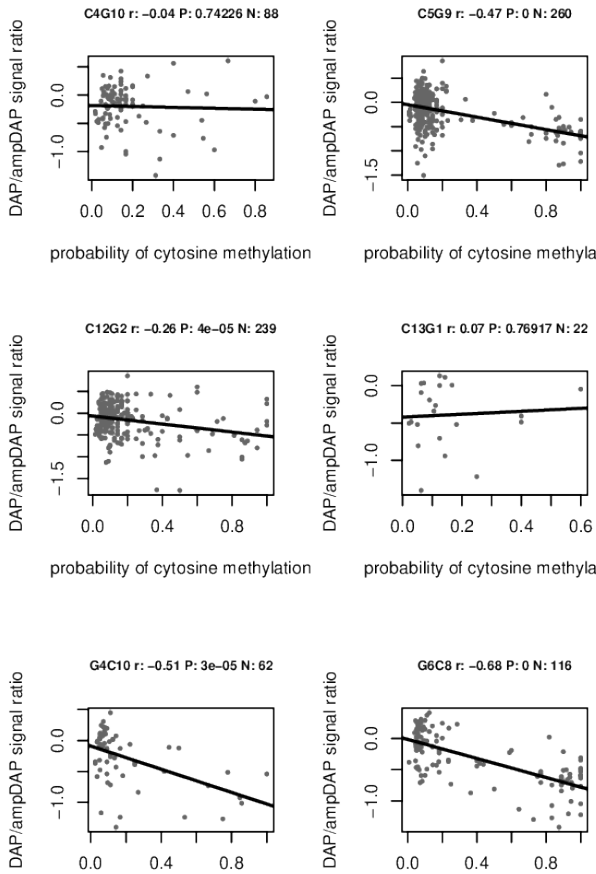

probability of cytosine methylation

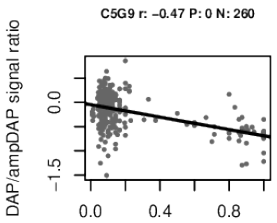

probability of cytosine methylation

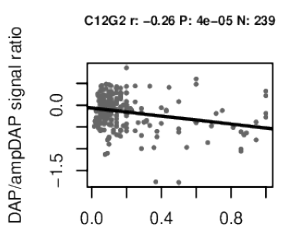

probability of cytosine methylation

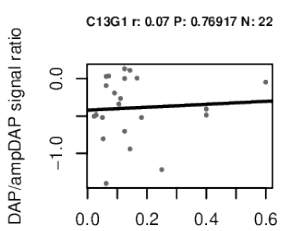

probability of cytosine methylation

probability of cytosine methylatio

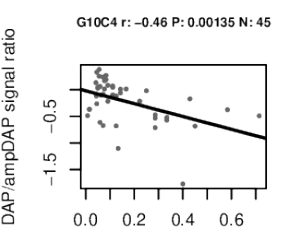

probability of cytosine methylation

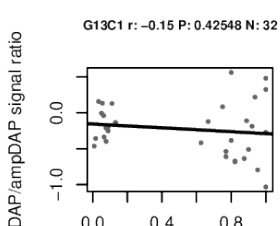

probability of cytosine methylation

861

862 Supplementary Figure 4: Effect of methylation of each individual position on ERF017

863 binding.

864 Relation between methylation probability at a single site in the predicted best binding site

865 and the $\log _{10}$-scaled relative binding site intensity of a DAP-seq versus an ampDAP-seq

866 experiment for ERF017 (At1g19210) on the forward (A) and reverse (B) strand. 


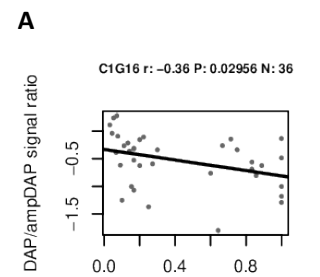

probability of cytosine methylatio

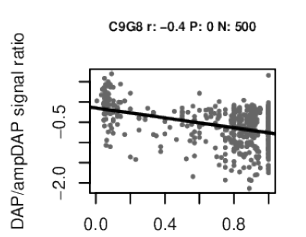

probability of cytosine methylation

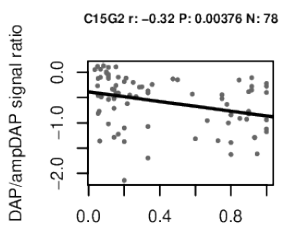

probability of cytosine methylation

870

871

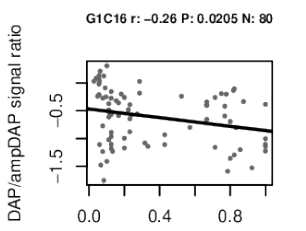

probability of cytosine methylation

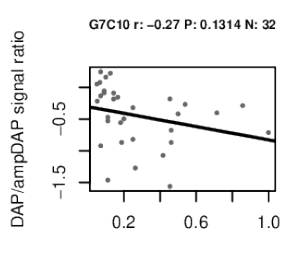

probability of cytosine methylation

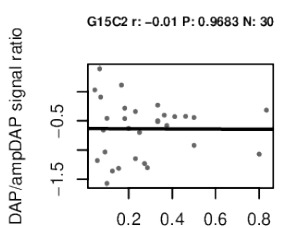

probability of cytosine methylation
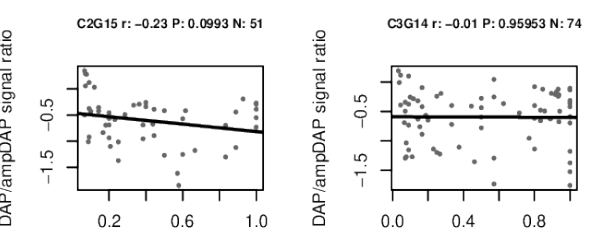

probability of cytosine methylation

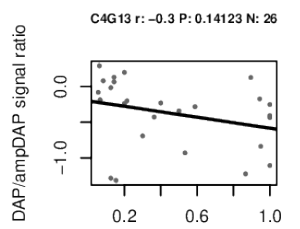

probability of cytosine methylation

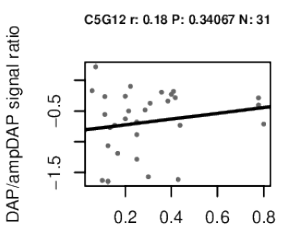

probability of cytosine methylation

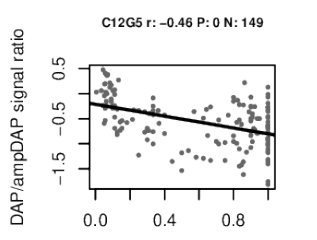

probability of cytosine methylation

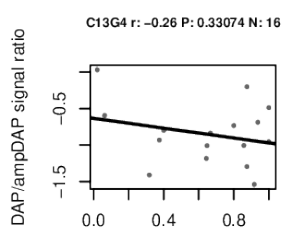

probability of cytosine methylation

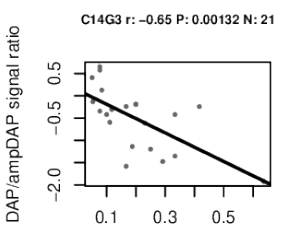

probability of cytosine methylation

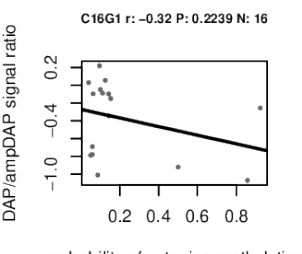

probability of cytosine methylation
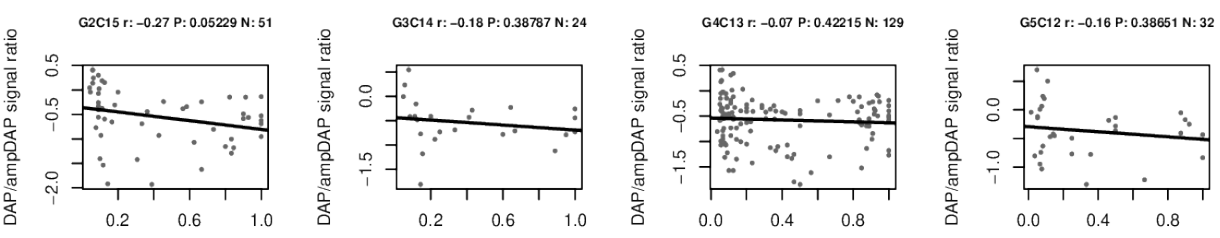

probability of cytosine methylation

probability of cytosine methylation

probability of cytosine methylation

probability of cytosine methylation
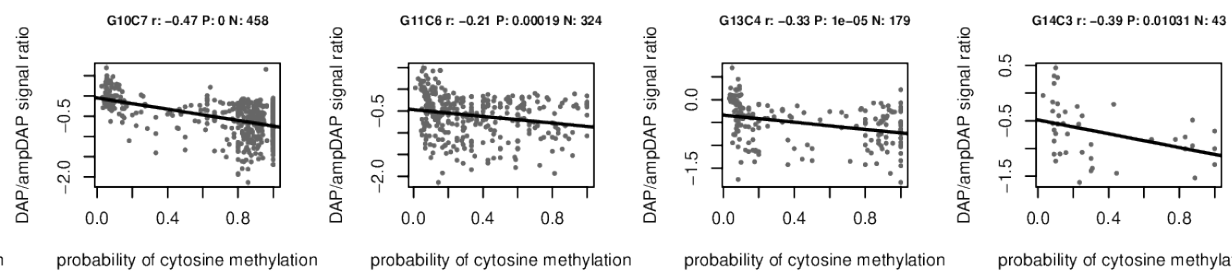

probability of cytosine methylation

probability of cytosine methylation

probability of cytosine methylation

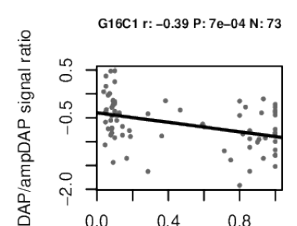

probability of cytosine methylation

873 Supplementary Figure 5: Effect of methylation of each individual position on trihelix-

874 domain containing protein (AT3G10030) binding.

875 Relation between methylation probability at a single site in the predicted best binding site

876 and the $\log _{10}$-scaled relative binding site intensity of a DAP-seq versus an ampDAP-seq

877 experiment for trihelix-domain containing protein (AT3G10030) on the forward (A) and

878 reverse (B) strand. 
A

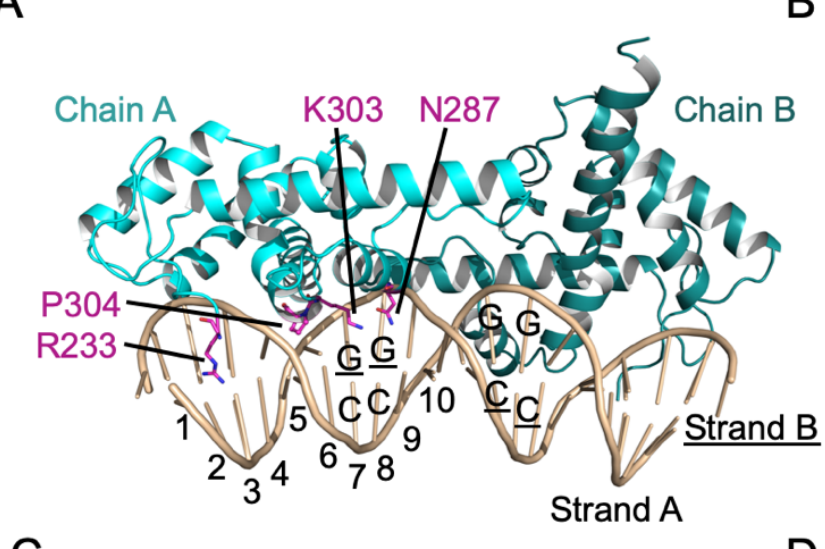

C

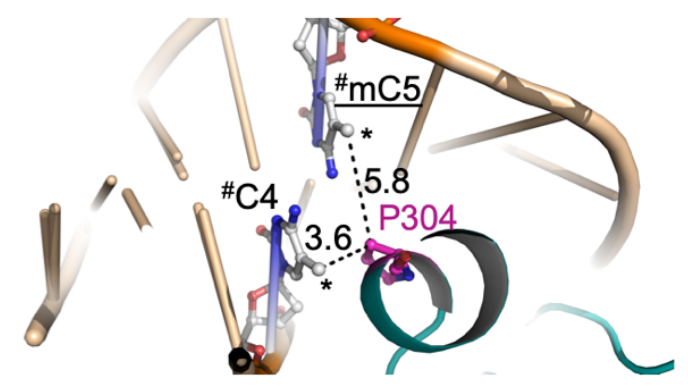

B
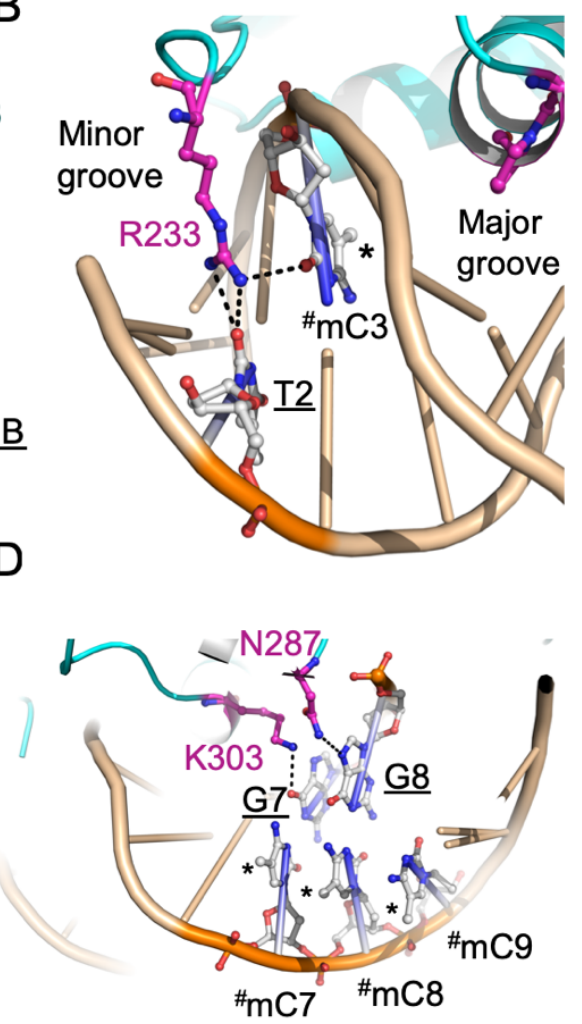

879

880

881

882

883

884

885

886

887

888

889

890

891

892

893

894

895

896

Supplementary Figure 6. Structural features suggesting that LFY is intrinsically only mildly sensitive to cytosine methylation.

(A) Overview of LFY-DBD dimer (Chain A and B) bound to DNA sequence from AP1 promoter (PDB 2vy1); Residues involved in base readout are highlighted in pink. LFY binding sequence is pseudo-palindromic and composed of two half-sites. One half-site is numbered from 1 to 10 with position 10 being the center of the pseudo-palindrome. The two DNA strands are designated as strand $A$ and strand $B$ (underlined), respectively. The highly conserved CG base pairs in both strands are annotated (bases from strand $B$ are underlined). (B) Base readout residue R233 interacts with bases from position 2 and 3 in the minor groove. C3 is mutated to methylated cytosine, designated as "mC3 (\# stands for mutation). Its methyl group facing the major groove, thus would not interfere with R233 base readout interactions. (C) Methylation of cytosine in position 4 (strand A) or position 5 (strand B) helps LFY binding by forming hydrophobic interaction between methyl groups and $C \beta$ of $P 308$; The methyl groups are in close proximity to P304. Distances are measured based on mutation done in the model of PDB 2vy1. (D) C7, C8, and C9 are mutated to mCs. Their methyl groups are distant from N287 and $\mathrm{K} 303(>8 \AA)$, thus are unlikely to interfere with the base readout interactions. The base readout interactions are indicated by black dash lines. Methyl groups are indicated by *. A base marked 
897 with \# stands for a mutated base relative to the native structure. It has to be noted that the 898 methylation pattern in the figure is to visualize their relative position to key base readout 899 residues, they might not occur in vivo, e.g., the three continuum $\mathrm{mC}$ in (D).

900 

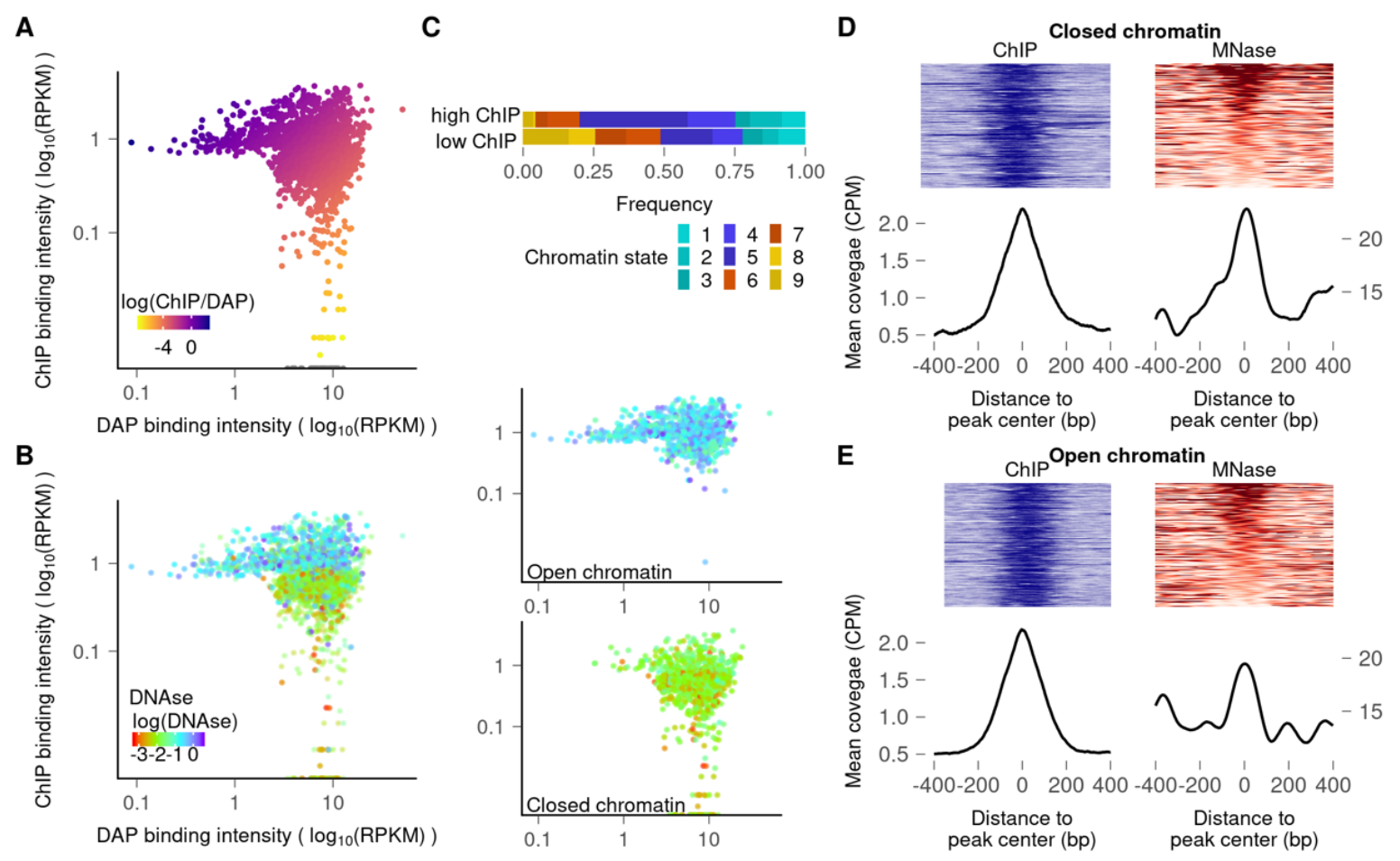

901

902 Supplementary Figure 7: Interaction between LFY and closed chromatin regions in

903 inflorescence tissues

904 (A) Plots comparing the LFY binding intensities in ChIP-seq (inflorescence tissue of 35S::LFY-

905 GR ap1 cal; Goslin et al., 2017) vs DAP-seq experiments. The heatmap is based on the ChIP-

906 seq/DAP-seq intensity ratio. (B) Overlay of DNasel signal (heat map) on LFY bound regions,

907 with DAP-seq (X-axis) and ChIP-seq (Y-axis) peak coverages. The two panels on the right show

908 the same regions split into open (upper panel) and closed (lower panel) chromatin states. (C)

909 Distribution of chromatin states 1 to 9 according to (Sequeira-Mendes et al., 2014) for the first

910 and last decile of LFY bound regions based on the ChIP-seq signal. (D-E) MNase signal around

911 ChIP-seq peak centers in closed (D) or open (E) chromatin regions. Upper panels show ChIP-

912 seq and MNase-seq coverage for each peak ordered based on the MNase-seq signal. Lower

913 panels represent the mean coverage.

914

915

916 
$-15$
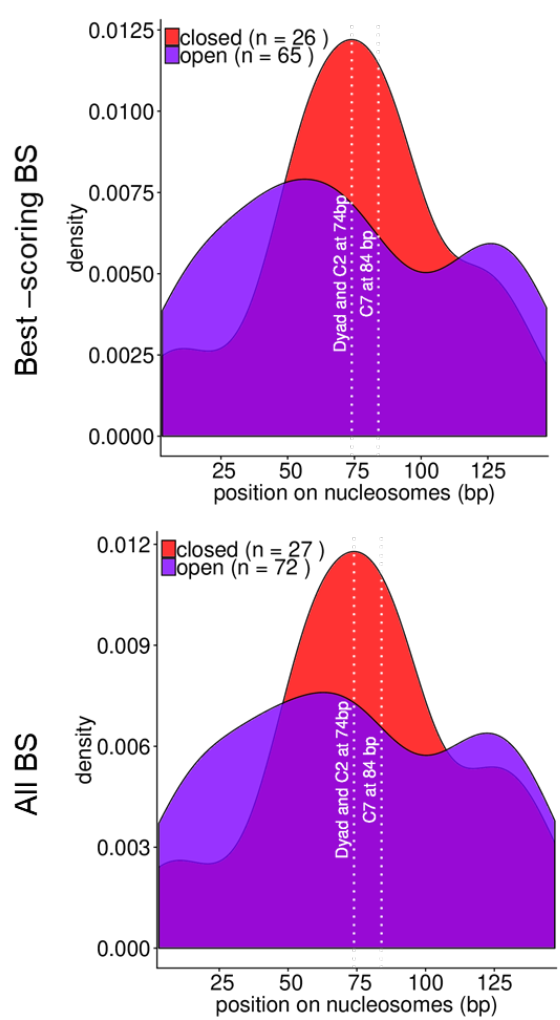

$-20$
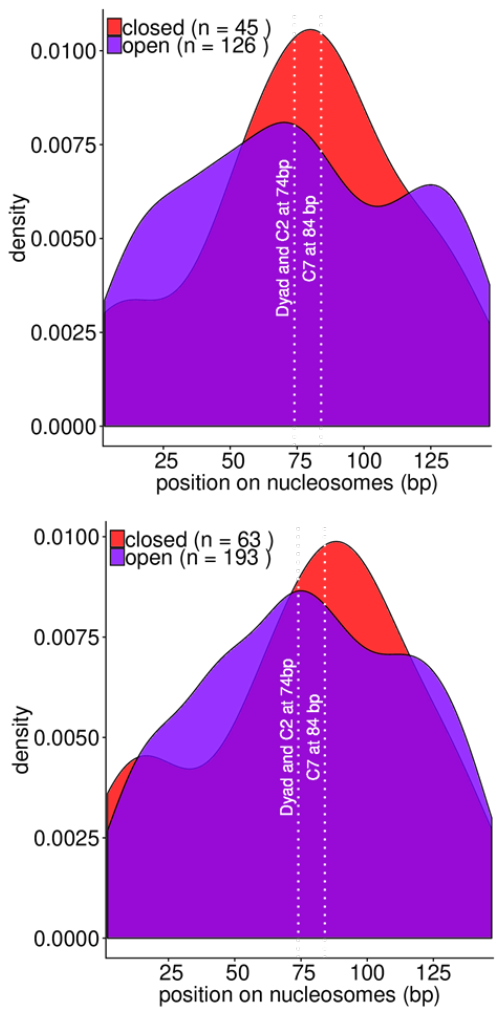

$-25$
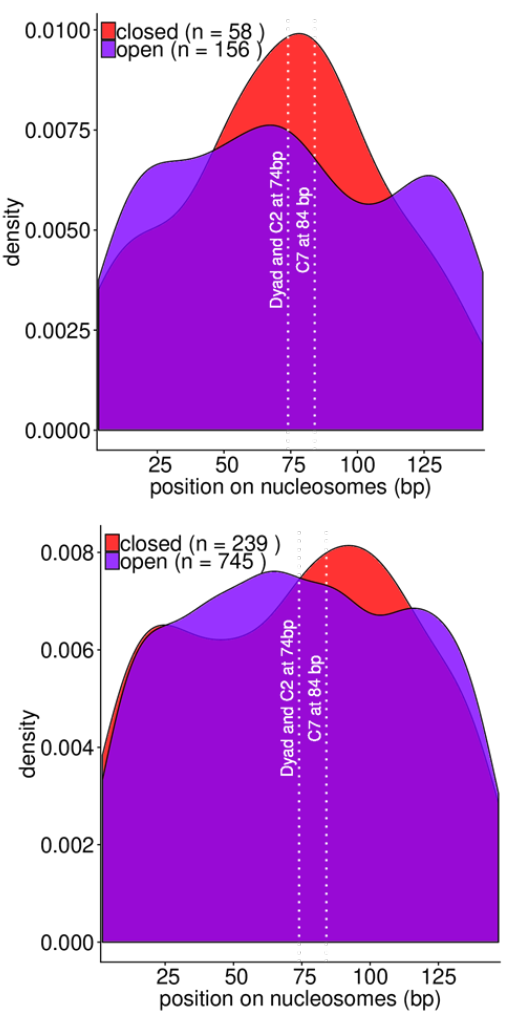

918 Supplementary Figure 8: Density plots of LFY binding sites

(BS) central position on

919 nucleosomes in open and closed chromatin for flower tissues.

920 Plots are shown for BS with a score greater than -15 (left), -20 (middle), and -25 (right), for 921 best-scoring BS only (top), and all BS above the score threshold (bottom). 

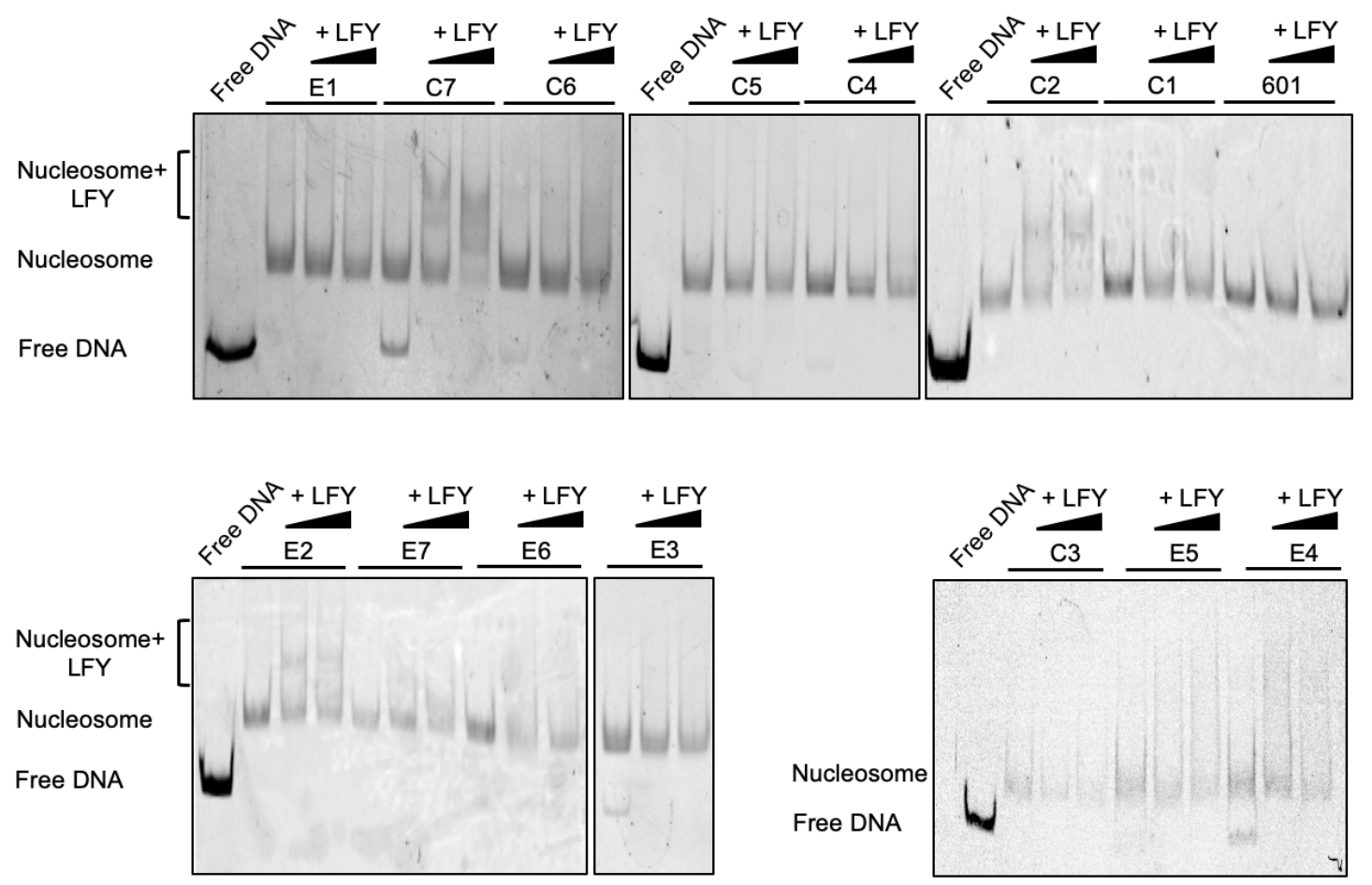

924 Supplementary Figure 9: LFY nucleosomal DNA binding assayed by EMSA using 601 925 sequences with a LFYBS inserted at different positions.

926 The positions of LFYBS in the 601 sequence are illustrated in Figure 3B and their sequences 927 are given in Table S2. Two concentrations of LFY ( $250 \mathrm{nM}$ and $1 \mu \mathrm{M}$ ) were used. LFY binds to 928 nucleosomes with a LFYBS at C2 and C7 positions; There is also weak binding at E2 but it is 929 weak and less reproducible.

930 

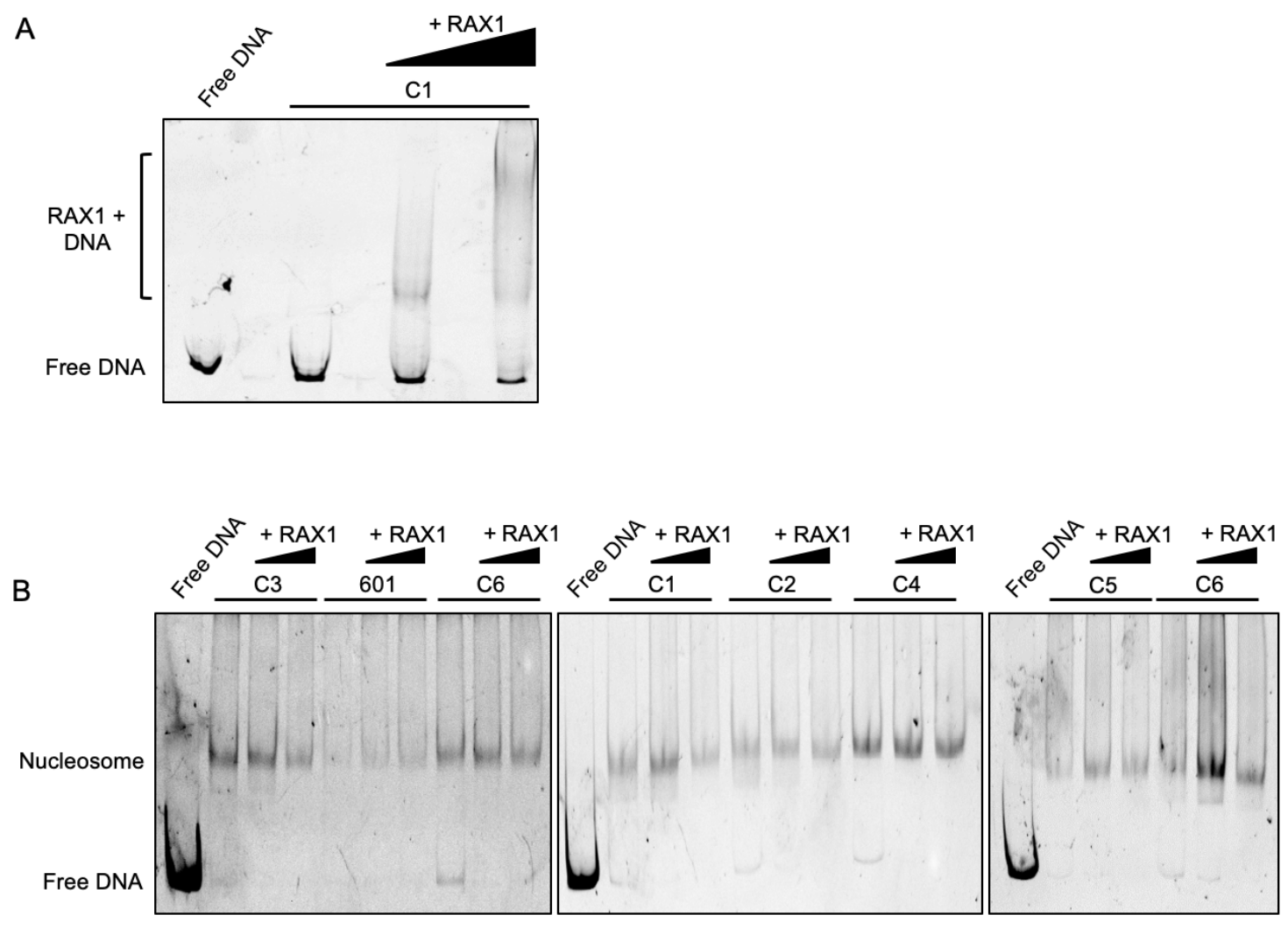

935 Supplementary Figure 10: EMSA testing RAX1 nucleosomal DNA binding on 601 sequence

936 with a RAX binding site inserted at different positions.

937 (A) EMSA with different concentrations of RAX1 (250 nM and $1 \mu \mathrm{M})$ and free 601 DNA with a

938 RAX1 binding site showing that recombinant RAX1 protein is active. (B) EMSA with RAX1 (250

$939 \mathrm{nM}$ and $1 \mu \mathrm{M}$ ) and 601 nucleosomes without or with a RAX1 binding site inserted at different

940 positions), showing that RAX1 has no nucleosomal DNA binding activity. RAX1 binding site

941 insertion in 601 sequences follows the same principle as for LFYBS illustrated in Figure 3B.

942 DNA sequences are given in Table S3.

943

944 


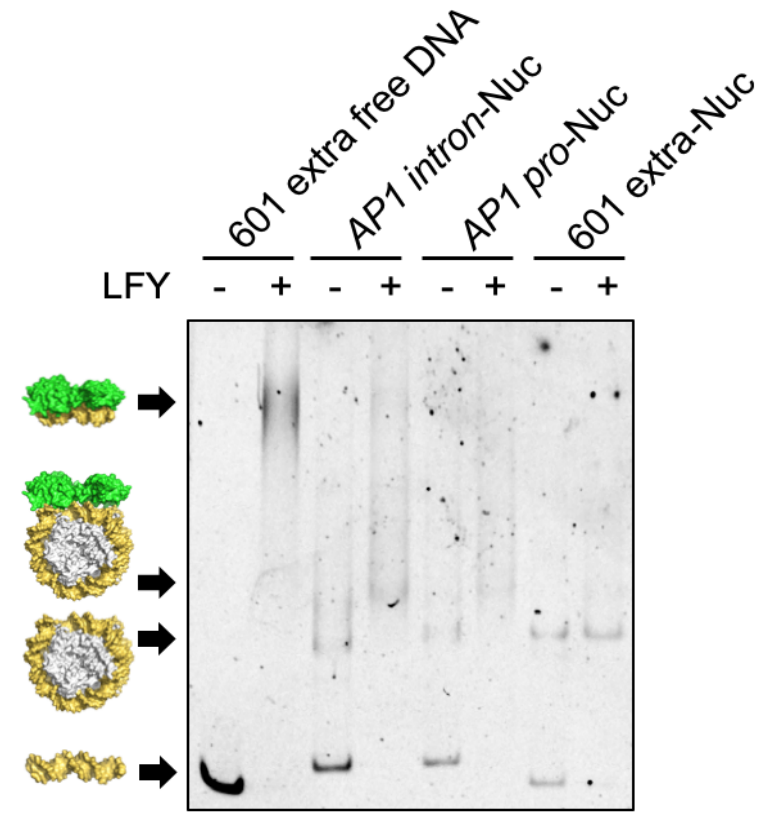

946

947 Supplementary Figure 11: LFY binds nucleosomes assembled with AP1 intron and promoter 948 sequences (related to Figure 3E).

949 Free DNA or reconstructed nucleosomes are indicated on top of the lanes. The mention extra 950 indicates that the sequence used is longer due to the presence of the amplification primers 951 used for $A P 1$ sequences (see table 2). The last two lanes are controls showing that these extra 952 sequences do not bind to LFY. Arrows and drawings on the side indicate from bottom to up 953 are free DNA, nucleosome alone, LFY nucleosome complex, and free DNA LFY complex. 


\section{Supplementary Table 1.}

957 Mapping and peak calling statistics of DAP-seq and ampDAP-seq experiments

\begin{tabular}{|c|c|c|c|c|c|c|c|c|c|c|c|c|c|c|}
\hline Study & $\begin{array}{c}\text { Transcription } \\
\text { factor }\end{array}$ & Library & DNA & \begin{tabular}{|l|} 
Read \\
type
\end{tabular} & $\begin{array}{l}\text { \#Raw } \\
\text { reads }\end{array}$ & $\begin{array}{l}\text { IS } \\
\text { (bp) }\end{array}$ & $\begin{array}{c}\text { \#Mapped } \\
\text { reads }\end{array}$ & $\begin{array}{l}\text { Mappi } \\
\text { ng rate } \\
(\%)\end{array}$ & $\begin{array}{c}\text { \#Filtered } \\
\text { reads }\end{array}$ & $\begin{array}{c}\text { \#Peaks } \\
\text { (MACS2) }\end{array}$ & $\begin{array}{c}\# \text { Consensus } \\
\text { peaks } \\
\text { (MSPC) }\end{array}$ & $\begin{array}{l}\text { Filtered } \\
\text { peaks }\end{array}$ & $\begin{array}{c}\# R C \text { in } \\
\text { filtered } \\
\text { peaks }\end{array}$ & FRIP \\
\hline \multirow{7}{*}{ This work } & Control & LIB1-1 & $\begin{array}{c}\text { Ampli } \\
\text { fied }\end{array}$ & PE & 22535270 & 249 & 10850451 & 48 & 9449616 & NA & NA & NA & NA & NA \\
\hline & \multirow{6}{*}{$\begin{array}{c}\text { LFY } \\
\text { (AT5G61850) }\end{array}$} & LIB11-13 & \multirow{3}{*}{$\begin{array}{c}\text { Ampli } \\
\text { fied }\end{array}$} & PE & 21094694 & 171 & 7515152 & 36 & 6266961 & 8692 & \multirow{3}{*}{4791} & \multirow{6}{*}{9116} & $\begin{array}{c}146722 \\
6\end{array}$ & 0.23 \\
\hline & & LIB11-14 & & $\mathrm{PE}$ & 16661008 & 194 & 6005517 & 36 & 5059651 & 7656 & & & $\begin{array}{c}114554 \\
6\end{array}$ & 0.23 \\
\hline & & LIB11-15 & & PE & 15667010 & 191 & 5132935 & 33 & 4480885 & 5553 & & & \begin{tabular}{|l|}
835042 \\
\end{tabular} & 0.19 \\
\hline & & 2 LIB11-20 & \multirow{3}{*}{$\begin{array}{c}\text { Non } \\
\text { ampli } \\
\text { fied }\end{array}$} & PE & 74147498 & 170 & 32781695 & 44 & 20940972 & 29365 & \multirow{3}{*}{20760} & & 747496 & 0.36 \\
\hline & & 2LIB11-21 & & PE & 42482466 & 180 & 18406436 & 43 & 13795444 & 29641 & & & $\begin{array}{c}420823 \\
2\end{array}$ & 0.31 \\
\hline & & 2LIB11-22 & & PE & 29556830 & 203 & 13689955 & 46 & 10729125 & 29910 & & & $\begin{array}{c}313440 \\
3\end{array}$ & 0.29 \\
\hline \multirow{5}{*}{$\begin{array}{c}\text { Re- } \\
\text { mapping } \\
\text { of } \\
\text { O'Malley } \\
\text { et al } 2016\end{array}$} & Control & SRR2926068 & \begin{tabular}{|c|} 
Non \\
ampli \\
fied
\end{tabular} & PE & 8482778 & NA & 4002772 & 47 & 586857 & NA & NA & NA & NA & NA \\
\hline & \multirow{2}{*}{$\begin{array}{c}\text { ERF017 } \\
\text { (AT1G19210) }\end{array}$} & SRX1412012 & $\begin{array}{c}\text { Ampli } \\
\text { fied }\end{array}$ & SE & 3628476 & NA & 1432383 & 39 & 1432383 & 4786 & NA & \multirow[t]{2}{*}{5266} & 253696 & 0.18 \\
\hline & & SRX1412013 & \begin{tabular}{|c|} 
Non \\
ampli \\
fied
\end{tabular} & SE & 8258696 & NA & 674974 & 8 & 674974 & 8959 & NA & & 428658 & 0.64 \\
\hline & \multirow{2}{*}{$\begin{array}{c}\text { Trihelix } \\
\text { (AT3G10030) }\end{array}$} & SRX1412646 & $\begin{array}{c}\text { Ampli } \\
\text { fied }\end{array}$ & SE & 1327104 & NA & 490155 & 37 & 490155 & 1762 & NA & \multirow[t]{2}{*}{1653} & 90280 & 0.18 \\
\hline & & SRX1412647 & \begin{tabular}{|c|} 
Non \\
ampli \\
fied
\end{tabular} & $\mathrm{SE}$ & 998448 & NA & 352811 & 35 & 352811 & 806 & NA & & 88665 & 0.25 \\
\hline & \multicolumn{14}{|c|}{$\begin{array}{l}\text { *Peaks with RPKM>3 (all transcription factors) and maximum height greater than } 10 \text { (LFY) in the consensus DAP-seq or amplified DAP-seq } \\
\text { experiment were merged. PE, paired-end. SE, single-end. IS, mean insert size. FRIP, the fraction of filtered reads in peaks. NA, not } \\
\text { applicable. }\end{array}$} \\
\hline
\end{tabular}




\section{Supplementary Table 2.}

962 DNA sequences used for nucleosome reconstruction with LFY binding site underlined 963

\begin{tabular}{|c|c|}
\hline $\begin{array}{l}\text { DESCRIPTIO } \\
\qquad \mathrm{N}\end{array}$ & \\
\hline $\begin{array}{l}\text { Widom } 601 \\
\text { sequence }\end{array}$ & $\begin{array}{l}\text { ATCGATGTATATATCTGACACGTGCCTGGAGACTAGGGAGTAATCCCCTTGGCGGTTAAAACGCGGGGGACAG } \\
\text { CGCGTACGTGCGTTTAAGCGGTGCTAGAGCTGTCTACGACCAATTGAGCGGCCTCGGCACCGGGATTCTGAT }\end{array}$ \\
\hline $\begin{array}{l}\text { LFY-C1 (LFY } \\
\text { binding site } \\
\text { is } \\
\text { underlined) }\end{array}$ & $\begin{array}{l}\text { ATCGATGTATATATCTGACACGTGCCTGGAGACTAGGGAGTAATCCCCTTGGCGGTTAAAACATTGACCAgCG } \\
\text { GGTAATTGTGCGTTTAAGCGGTGCTAGAGCTGTCTACGACCAATTGAGCGGCCTCGGCACCGGGATTCTGAT }\end{array}$ \\
\hline LFY-C2 & $\begin{array}{l}\text { ATCGATGTATATATCTGACACGTGCCTGGAGACTAGGGAGTAATCCCCTTGGCGGTTAAAACGCATTGACCAg } \\
\text { CGGGTAATTGCGTTTAAGCGGTGCTAGAGCTGTCTACGACCAATTGAGCGGCCTCGGCACCGGGATTCTGAT }\end{array}$ \\
\hline LFY-C3 & $\begin{array}{l}\text { ATCGATGTATATATCTGACACGTGCCTGGAGACTAGGGAGTAATCCCCTTGGCGGTTAAAACGCGCATTGACC } \\
\text { AgCGGGTAATTGTTTAAGCGGTGCTAGAGCTGTCTACGACCAATTGAGCGGCCTCGGCACCGGGATTCTGAT }\end{array}$ \\
\hline LFY & $\begin{array}{l}\text { ATCGATGTATATATCTGACACGTGCCTGGAGACTAGGGAGTAATCCCCTTGGCGGTTAAAACGCGGGCATTGA } \\
\text { CCAgCGGGTAATTGTAAGCGGTGCTAGAGCTGTCTACGACCAATTGAGCGGCCTCGGCACCGGGATTCTGAT }\end{array}$ \\
\hline LFY & $\begin{array}{l}\text { ATCGATGTATATATCTGACACGTGCCTGGAGACTAGGGAGTAATCCCCTTGGCGGTTAAAACGCGGGGGCATT } \\
\text { GACCAgCGGGTAATTGAGCGGTGCTAGAGCTGTCTACGACCAATTGAGCGGCCTCGGCACCGGGATTCTGAT }\end{array}$ \\
\hline LFY-C6 & $\begin{array}{l}\text { ATCGATGTATATATCTGACACGTGCCTGGAGACTAGGGAGTAATCCCCTTGGCGGTTAAAACGCGGGGGACCA } \\
\text { TTGACCAgCGGGTAATTGCGGTGCTAGAGCTGTCTACGACCAATTGAGCGGCCTCGGCACCGGGATTCTGAT }\end{array}$ \\
\hline & $\begin{array}{l}\text { ATCGATGTATATATCTGACACGTGCCTGGAGACTAGGGAGTAATCCCCTTGGCGGTTAAAACGCGGGGGACAG } \\
\text { САTTGACCAgCGGGTAATTGGTGCTAGAGCTGTCTACGACCAATTGAGCGGCCTCGGCACCGGGATTCTGAT }\end{array}$ \\
\hline LFY & $\begin{array}{l}\text { ATCGATGTATATATCTGACACGTGCCTGGAGACTAGGGAGTAATCCCCTTGGCGGTTAAAACGCGGGGGACAG } \\
\text { CGCGTACGCATTGACCAgCGGGTAATTGTCTGTCTACGACCAATTGAGCGGCCTCGGCACCGGGATTCTGAT }\end{array}$ \\
\hline LFY & $\begin{array}{l}\text { ATCGATGTATATATCTGACACGTGCCTGGAGACTAGGGAGTAATCCCCTTGGCGGTTAAAACGCGGGGGACAG } \\
\text { CGCGTACGTGCATTGACCAgCGGGTAATTGTGTCTACGACCAATTGAGCGGCCTCGGCACCGGGATTCTGAT }\end{array}$ \\
\hline LFY-E3 & $\begin{array}{l}\text { ATCGATGTATATATCTGACACGTGCCTGGAGACTAGGGAGTAATCCCCTTGGCGGTTAAAACGCGGGGGACAG } \\
\text { CGCGTACGTGCGCATTGACCAgCGGGTAATTGTCTACGACCAATTGAGCGGCCTCGGCACCGGGATTCTGAT }\end{array}$ \\
\hline LFY-E4 & $\begin{array}{l}\text { ATCGATGTATATATCTGACACGTGCCTGGAGACTAGGGAGTAATCCCCTTGGCGGTTAAAACGCGGGGGACAG } \\
\text { CGCGTACGTGCGTTCATTGACCAgCGGGTAATTGTACGACCAATTGAGCGGCCTCGGCACCGGGATTCTGAT }\end{array}$ \\
\hline LFY-E5 & $\begin{array}{l}\text { ATCGATGTATATATCTGACACGTGCCTGGAGACTAGGGAGTAATCCCCTTGGCGGTTAAAACGCGGGGGACAG } \\
\text { CGCGTACGTGCGTTTACATTGACCAgCGGGTAATTGCGACCAATTGAGCGGCCTCGGCACCGGGATTCTGAT }\end{array}$ \\
\hline LFY-E6 & $\begin{array}{l}\text { ATCGATGTATATATCTGACACGTGCCTGGAGACTAGGGAGTAATCCCCTTGGCGGTTAAAACGCGGGGGACAG } \\
\text { CGCGTACGTGCGTTTAAGCATTGACCAgCGGGTAATTGACCAATTGAGCGGCCTCGGCACCGGGATTCTGAT }\end{array}$ \\
\hline LFY-E7 & $\begin{array}{l}\text { ATCGATGTATATATCTGACACGTGCCTGGAGACTAGGGAGTAATCCCCTTGGCGGTTAAAACGCGGGGGACAG } \\
\text { CGCGTACGTGCGTTTAAGCGCATTGACCAgCGGGTAATTGCAATTGAGCGGCCTCGGCACCGGGATTCTGAT }\end{array}$ \\
\hline$A P 1$ intron & $\begin{array}{l}\text { АTGAAAATAAACAATTTGATAAAAGAAAAAAAAAAAAAGAAGAACAGCTGTTGCTTGTTGGAGCTAAGTTTGA } \\
\text { CСATCGGTAAGAAGCCGATTTTAGGATGGAGTTAATTCTTTTTATGGATCCCCAGAGGTCAAAGACTCCCTAC } \\
\text { TCAGATTTGACATCTTTGTTTCAGTTTTAATTTCTAAAGTCTTCAGATTTTGTTTCGTAGA }\end{array}$ \\
\hline
\end{tabular}




\begin{tabular}{|l|l|}
\hline AP1 pro* & $\begin{array}{l}\text { ATGAAAATAAACAATTTGATACTTAAAAATATGAAAATAAACAATTTGATTATCGACGTCTCGTGAAGAGAAA } \\
\text { TGGGTAAGTAACATTGTACGGACCACTGGTCCTTCCCCAAGTGTCACCTTCGCTTTGCATTGACGGCGGAGAT } \\
\text { TTCCCTGTAGATCTACGAAACAAAATCTGAACCAACCAAAATTCAGATTTTGTTTCGTAGA }\end{array}$ \\
\hline $\mathbf{6 0 1}$ extra* & $\begin{array}{l}\text { ATGAAAATAAACAATTTGATATCGATGTATATATCTGACACGTGCCTGGAGACTAGGGAGTAATCCCCTTGGCGGTTA } \\
\text { AAACGCGGGGGACAGCGCGTACGTGCGTTTAAGCGGTGCTAGAGCTGTCTACGACCAATTGAGCGGCCTCGGCACCG } \\
\\
\text { GGATTCTGATTTCAGATTTTTTTCGTAGA }\end{array}$ \\
\hline
\end{tabular}

* indicates sequences for nucleosome reconstruction with extended 5' and 3' ends (italicized)

965 originating from amplification primers. 
966 Supplementary Table 3. DNA sequences used for nucleosome reconstruction

967 with RAX1 binding site underlined

968

\begin{tabular}{|c|c|}
\hline DESCRIPTION & SEQUENCE \\
\hline $\begin{array}{l}\text { RAX1-C1 (RAX1 } \\
\text { binding site is } \\
\text { underlined) }\end{array}$ & $\begin{array}{l}\text { ATCGATGTATATATCTGACACGTGCCTGGAGACTAGGGAGTAATCCCCTTGGCGGTTAAAATTGGGTACCTAACTTTCT } \\
\text { AATGCGTTTAAGCGGTGCTAGAGCTGTCTACGACCAATTGAGCGGCCTCGGCACCGGGATTCTGAT }\end{array}$ \\
\hline RAX1-C2 & $\begin{array}{l}\text { ATCGATGTATATATCTGACACGTGCCTGGAGACTAGGGAGTAATCCCCTTGGCGGTTAAAACGTTGGGTACCTAACTTT } \\
\text { СTAACGTTTAAGCGGTGCTAGAGCTGTCTACGACCAATTGAGCGGCCTCGGCACCGGGATTCTGAT }\end{array}$ \\
\hline RAX1-C3 & $\begin{array}{l}\text { ATCGATGTATATATCTGACACGTGCCTGGAGACTAGGGAGTAATCCCCTTGGCGGTTAAAACGCGTTGGGTACCTAACT } \\
\text { TTCTAATTTAAGCGGTGCTAGAGCTGTCTACGACCAATTGAGCGGCCTCGGCACCGGGATTCTGAT }\end{array}$ \\
\hline RAX1-C4 & $\begin{array}{l}\text { ATCGATGTATATATCTGACACGTGCCTGGAGACTAGGGAGTAATCCCCTTGGCGGTTAAAACGCGGGTTGGGTACCTAA } \\
\text { СTTTCTAATAAGCGGTGCTAGAGCTGTCTACGACCAATTGAGCGGCCTCGGCACCGGGATTCTGAT }\end{array}$ \\
\hline RAX1-C5 & $\begin{array}{l}\text { ATCGATGTATATATCTGACACGTGCCTGGAGACTAGGGAGTAATCCCCTTGGCGGTTAAAACGCGGGGGTTGGGTACCT } \\
\text { AACTTTCTAAAGCGGTGCTAGAGCTGTCTACGACCAATTGAGCGGCCTCGGCACCGGGATTCTGAT }\end{array}$ \\
\hline RAX1-C6 & $\begin{array}{l}\text { ATCGATGTATATATCTGACACGTGCCTGGAGACTAGGGAGTAATCCCCTTGGCGGTTAAAACGCGGGGGACTTGGGTAC } \\
\text { СTAACTTTCTAACGGTGCTAGAGCTGTCTACGACCAATTGAGCGGCCTCGGCACCGGGATTCTGAT }\end{array}$ \\
\hline RAX1-C7 & $\begin{array}{l}\text { ATCGATGTATATATCTGACACGTGCCTGGAGACTAGGGAGTAATCCCCTTGGCGGTTAAAACGCGGGGGACAGTTGGGT } \\
\text { ACCTAACTTTCTAAGTGCTAGAGCTGTCTACGACCAATTGAGCGGCCTCGGCACCGGGATTCTGAT }\end{array}$ \\
\hline
\end{tabular}

969

970 